\title{
Taming Displayed Tense Logics Using Nested Sequents With Deep Inference
}

\author{
Rajeev Goré, Linda Postniece, and Alwen Tiu \\ Logic and Computation Group \\ College of Engineering and Computer Science \\ The Australian National University
}

\begin{abstract}
We consider two sequent calculi for tense logic in which the syntactic judgements are nested sequents, i.e., a tree of traditional onesided sequents built from multisets of formulae. Our first calculus SKt is a variant of Kashima's calculus for Kt, which can also be seen as a display calculus, and uses "shallow" inference whereby inference rules are only applied to the top-level nodes in the nested structures. The rules of $\mathbf{S K t}$ include certain structural rules, called "display postulates", which are used to bring a node to the top level and thus in effect allow inference rules to be applied to an arbitrary node in a nested sequent. The cut elimination proof for SKt uses a proof substitution technique similar to that used in cut elimination for display logics. We then consider another, more natural, calculus DKt which contains no structural rules (and no display postulates), but which uses deep-inference to apply inference rules directly at any node in a nested sequent. This calculus corresponds to Kashima's $S 2 K t$, but with all structural rules absorbed into logical rules. We show that SKt and DKt are equivalent, that is, any cut-free proof of SKt can be transformed into a cut-free proof of $\mathbf{D K t}$, and vice versa. We consider two extensions of tense logic, Kt.S4 and $S 5$, and show that this equivalence between shallow- and deep-sequent systems also holds. Since deep-sequent systems contain no structural rules, proof search in the calculi is easier than in the shallow calculi. We outline such a procedure for the deep-sequent system DKt and its $S 4$ extension.
\end{abstract}

\section{Introduction}

Belnap's Display Logic [2] (we prefer the term display calculi) is an extremely general proof-theoretical framework with the property that any sequent containing a particular formula occurrence $A$ can be transformed into another sequent in which the occurrence of $A$ is either the whole of the antecedent or the whole of the succedent, using only a subset of the rules called the display postulates. The occurrence of $A$ is then said to be displayed. The most pleasing property of display calculi however is that if the rules of the display calculus enjoy eight easily checked conditions, then the calculus is guaranteed to obey cut-admissibility. That is, one single cut-admissibility proof suffices for all display calculi. This modularity makes it an excellent framework for designing sequent calculi for 
logics, particularly when we wish to mix and match the intuitionistic, modal, or substructural aspects of different logics into a new logic $[17,7,6]$.

The generality of display calculi is obtained by adding a structural proxy for every logical connective and using residuation principles to implement the display property. For example, a display calculus for classical propositional logic usually contains Gentzen's "comma", but also a unary involutive structural connective "star" which allows us to flip structures from right/left to left/right of turnstile.

The main disadvantage of display calculi is that the display postulates can and must create large structures during the process of displaying a particular formula occurrence, making display calculi bad for backward proof-search. Display calculi also typically contain an explicit rule of contraction which duplicates complex structures when applied backwards, making it even harder to use them for backward proof search. A disciplined proof-theoretic methodology for transforming a display calculus into a more manageable traditional "contraction-free" calculus whilst preserving cut-admissiblity is therefore an important goal.

Our first step towards taming display calculi is to limit the structural connectives used in the calculi and consequently, the number of display postulates. Specifically, we work within display structures which can be viewed as a tree of traditional Gentzen's sequents, called nested sequents, which have been used previously by Kashima [12] and, independently, by Brünnler [3,4] to present several modal and tense logics. As in display calculi, Kashima's nested-sequent calculi contain "display-like" rules, called the turn rules in [12] and residuation rules in the display logic literature, which can be seen as tree transformations to bring a node in the nested sequent to the root. These residuation rules, and their interaction with structure contraction, are largely responsible for the difficulty in finding a proof search procedure for display-like calculi. Our second step is therefore to eliminate these rules without losing completeness.

We use Kashima's calculi for tense logics as a starting point for our proof theoretic (as opposed to the model-theoretic approach of Kashima) investigation into the broader problem of taming display calculi for proof search. We have recently shown that it is possible to tame the display calculus for Bi-Intuitionistic logic [8] by using nested sequents with a limited display property. The resulting calculus, $L B i I_{1} t_{1}$, still enjoys cut-admissibility. However, proof search for LBiInt $t_{1}$ still suffers essentially the same problem as in display calculi, due to the presence of residuation and contraction on structures. In the same paper, we also show that these two problems can be eliminated entirely by a derived calculus LBiInt $_{2}$. However, the completeness proof of LBiInt 2 w.r.t. LBiInt $t_{1}$ was done via a detour through a third calculus GBiInt which is known to be semantically complete, and it was not clear how this methodology could be generalised to arbitrary display calculi for which the semantics may be unknown.

Here, we show that for some classical tense logics, residuation, seen as treetransformations on nested sequents, and contraction (on general structures) are admissible if we allow a more liberal form of inference rule. Traditional rules of Gentzen's sequent calculus and display calculi apply only to formulae on the top level of a nested sequent. We shall call these rules "shallow inference". 
Residuation and contraction become admissible once we allow deep inference, the ability to apply inference rules at any depth in a nested sequent.

The choice of classical tense logics as a case study is convenient because nested sequent calculi for these logics have already been given by Kashima [12]. But as we have noted earlier, Kashima's work is semantic based as there is no syntactic cut elimination procedure in his work. Thus our work is the first which shows direct syntactic cut elimination for a nested-sequent calculus for tense logic, and also the first which establishes a direct correspondence between proofs in a display-like calculus (with explicit residuation rules) and proofs in a contraction-free deep-inference calculus (with no explicit residuation rules).

We begin with Kashima's first system SKt which contains structural connectives (proxies) for $\diamond$ and $\downarrow$ and contains explicit "turn" rules to capture the residuation conditions that hold between them. Kashima shows that SKt is sound with respect to the Kripke semantics for tense logic, but he does not prove cut-admissibility for this system. He instead gives another calculus S2Kt which allows rules to be applied at arbitrary depth, and shows that a sequent has a cut-free proof in SKt if it has a cut-free proof in S2Kt. In a second step, he shows that S2Kt minus cut is complete w.r.t. the Kripke semantics of tense logic, which together imply the completeness of SKt minus cut.

We first replace formula contraction with general contraction in Kashima's SKt, show that the resulting calculus enjoys a display property, and show that it also has cut-admissibility using an argument which is very similar to Belnap's cut-admissibility proof for display calculi. We then show that Kashima's S2Kt minus cut (in the form of our DKt) can be made contraction-free and that the display postulates of SKt are admissible in DKt, meaning that DKt can faithfully mimic cut-free SKt. We also show that SKt can mimic DKt by showing that all of the rules of DKt are actually derivable in SKt using the display property of SKt. We then show how to extend all these basic calculi to handle tense S4 and S5, but we are still not able to give a systematic method for converting the SKt-based calculi into the DKt-based calculi. Finally, we give a simple proof search strategy for DKt, as well as show how to add histories a là Heuerding to DS4 for terminating proof search in the tense logic Kt.S4.

Detailed proofs can be found in the appendix.

\section{Tense Logic}

To simplify presentation, we shall consider formulae of tense logic Kt which are in negation normal form (nnf), given by the following grammar:

$$
A:=a|\neg a| A \vee A|A \wedge A| \square A|\mathbf{\square} A| \diamond A \mid \diamond A .
$$

where $a$ ranges over atomic formulae and $\neg a$ is the negation of $a$. We shall denote with $\bar{A}$ the nnf of the negation of $A$. Implication can then be defined via negation: $A \rightarrow B=\bar{A} \vee B$. The axioms of minimal tense logic Kt are all the axioms of propositional logic, plus the following in their nnf form: 
$w \Vdash \neg A \quad$ iff $w \Vdash A$

$w \Vdash A \vee B$ iff $w \Vdash A$ or $w \Vdash B \quad w \Vdash A \wedge B$ iff $w \Vdash A$ and $w \Vdash B$

$w \Vdash \square A \quad$ iff $\forall u$. if $w R u$ then $u \Vdash A \quad w \Vdash \diamond A \quad$ iff $\exists u . w R u$ and $u \Vdash A$

$w \Vdash A \quad$ iff $\forall u$. if $u R w$ then $u \Vdash A \quad w \Vdash A \quad$ iff $\exists u . u R w$ and $u \Vdash A$

Fig. 1. Forcing of formulae

1. $A \rightarrow \square \backslash A=\bar{A} \vee \square \vee A$

2. $A \rightarrow \mathbf{\square} \diamond A=\bar{A} \vee \mathbf{\square} \vee A$

3. $\square(A \rightarrow B) \rightarrow(\square A \rightarrow \square B)=\diamond(A \wedge \bar{B}) \vee \diamond \bar{A} \vee \square B$

4. $(A \rightarrow B) \rightarrow(\boldsymbol{\square} \rightarrow \boldsymbol{\square} B)=\boldsymbol{\nabla}(A \wedge \bar{B}) \vee \wedge \bar{A} \vee \mathbf{\square}$.

The theorems of Kt are those that are generated from the above axioms and their substitution instances using the following rules:

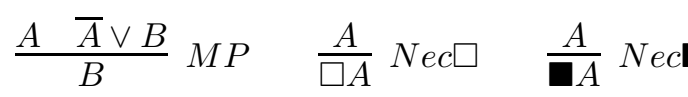

A $K t$-frame is a pair $\langle W, R\rangle$, with $W$ a non-empty set (of worlds) and $R \subseteq$ $W \times W$. A Kt-model is a triple $\langle W, R, V\rangle$, with $\langle W, R\rangle$ a Kt frame and $V$ : $A t m \rightarrow 2^{W}$ a valuation mapping each atom to the set of worlds where it is true.

For a world $w \in W$ and an atom $a \in A t m$, if $w \in V(a)$ then we write $w \Vdash a$ and say $a$ is forced at $w$; otherwise we write $w \forall a$ and say $a$ is rejected at $w$. Forcing and rejection of compound formulae is defined by mutual recursion in Figure 1. A Kt-formula $A$ is valid iff it is forced by all worlds in all models, i.e. iff $w \Vdash A$ for all $\langle W, R, V\rangle$ and for all $w \in W$.

\section{System SKt: a "shallow" calculus}

We consider a right-sided proof system for tense logic where the syntactic judgment is a tree of multisets of formulae, called a nested sequent. Nested sequents have been used previously in proof systems for modal and tense logics [12,3].

Definition 1. A nested sequent is a multiset

$$
\left\{A_{1}, \ldots, A_{k}, \circ\left\{\Gamma_{1}\right\}, \ldots, \circ\left\{\Gamma_{m}\right\}, \bullet\left\{\Delta_{1}\right\}, \ldots, \bullet\left\{\Delta_{n}\right\}\right\}
$$

where $k, m, n \geq 0$, and each $\Gamma_{i}$ and each $\Delta_{j}$ are themselves nested sequents.

We shall use the following notational conventions when writing nested sequents. We shall remove outermost braces, e.g., we write $A, B, C$ instead of $\{A, B, C\}$. Braces for sequents nested inside $\circ\{\}$ or $\bullet\{\}$ are also removed, e.g., instead of writing $\circ\{\{A, B, C\}\}$, we write $\circ\{A, B, C\}$. When we juxtapose two sequents, e.g., as in $\Gamma, \Delta$, we mean it is a sequent resulting from the multiset-union of $\Gamma$ and $\Delta$. When $\Delta$ is a singleton multiset, e.g., $\{A\}$ or $\left\{\circ\left\{\Delta^{\prime}\right\}\right\}$, we simply write: $\Gamma, A$ or $\Gamma, \circ\left\{\Delta^{\prime}\right\}$. Since we shall only be concerned with nested sequents, we shall refer to nested sequents simply as sequents in the rest of the paper. 


$$
\begin{aligned}
& \overline{\Gamma, a, \bar{a}} \text { id } \quad \frac{\Gamma, A \Delta, \bar{A}}{\Gamma, \Delta} \text { cut } \quad \frac{\Gamma, A \quad \Gamma, B}{\Gamma, A \wedge B} \wedge \quad \frac{\Gamma, A, B}{\Gamma, A \vee B} \vee \\
& \frac{\Gamma, \Delta, \Delta}{\Gamma, \Delta} \operatorname{ctr} \quad \frac{\Gamma}{\Gamma, \Delta} w k \quad \frac{\Gamma, \circ\{\Delta\}}{\bullet\{\Gamma\}, \Delta} r f \quad \frac{\Gamma, \bullet\{\Delta\}}{\circ\{\Gamma\}, \Delta} r p \\
& \frac{\Gamma, \bullet\{A\}}{\Gamma, \boldsymbol{\square}} \mathbf{\square} \quad \frac{\Gamma, \circ\{A\}}{\Gamma, \square A} \square \quad \frac{\Gamma, \bullet\{\Delta, A\}}{\Gamma, \bullet\{\Delta\}, \bullet A} \diamond \quad \frac{\Gamma, \circ\{\Delta, A\}}{\Gamma, \circ\{\Delta\}, \diamond A} \diamond
\end{aligned}
$$

Fig. 2. System SKt

The above definition of sequents can also be seen as a special case of structures in display calculi, e.g., with ',' (comma), • and o as structural connectives.

A context is a sequent with holes in place of formulae. A context with a single hole is written as $\Sigma[]$. Multiple-hole contexts are written as $\Sigma[] \cdots[]$, or abbreviated as $\Sigma^{k}[]$ where $k$ is the number of holes. We write $\Sigma^{k}[\Delta]$ to denote the sequent that results from filling the holes in $\Sigma^{k}[]$ uniformly with $\Delta$.

The shallow proof system for Kt, called SKt, is given in Figure 2. This is basically Kashima's system (also called SKt) [12], but with a more general contraction rule $(c t r)$, which allows contraction of arbitrary sequents. The modal fragment of SKt was also developed independently by Brünnler [3]. The general contraction rule is used to simplify our cut elimination proof, and as we shall see in Section 4, it can be replaced by formula contraction. System SKt can also be seen as a single-sided version of display calculus. The rules $r p$ and $r f$ are called the residuation rules. They are an example of display postulates commonly found in display calculus, and are used to bring a node in a nested sequent to the top level. The following is an analog of the display property of display calculus.

Proposition 1. Let $\Sigma[\Delta]$ be a sequent. Then there exists a sequent $\Gamma$ such that $\Sigma[\Delta]$ is derivable from $\Delta, \Gamma$ and vice versa, using only the rules rp and $r f$.

Soundness and completeness. To prove soundness, we first show that each sequent has a corresponding Kt-formula, and then show that the rules of SKt, reading them top down, preserves validity of the formula corresponding to the premise sequent. Completeness is shown by simulating Hilbert's system for tense logic in SKt. The translation from sequents to formulae are given below. In the translation, we assume two logical constants $\perp$ ('false') and $T$ ('true'). This is just a notational convenience, as the constants can be defined in a standard way, e.g., as $a \wedge \bar{a}$ and $a \vee \bar{a}$ for some fixed atomic proposition $a$. As usual, the empty disjunction denotes $\perp$ and the empty conjunction denotes $T$.

Definition 2. The function $\tau$ translates an $\mathbf{S K t}-$ sequent

$$
\left\{A_{1}, \ldots, A_{k}, \circ\left\{\Gamma_{1}\right\}, \ldots, \circ\left\{\Gamma_{m}\right\}, \bullet\left\{\Delta_{1}\right\}, \ldots, \bullet\left\{\Delta_{n}\right\}\right\}
$$

into the Kt-formula (modulo associativity and commutativity of $\vee$ and $\wedge$ ):

$$
A_{1} \vee \cdots \vee A_{k} \vee \square \tau\left(\Gamma_{1}\right) \vee \cdots \vee \square \tau\left(\Gamma_{m}\right) \vee \mathbf{\square} \tau\left(\Delta_{1}\right) \vee \cdots \vee \square \tau\left(\Delta_{n}\right)
$$

Theorem 1. A Kt-formula $A$ is valid iff $A$ is $\mathbf{S K t}$-derivable. 


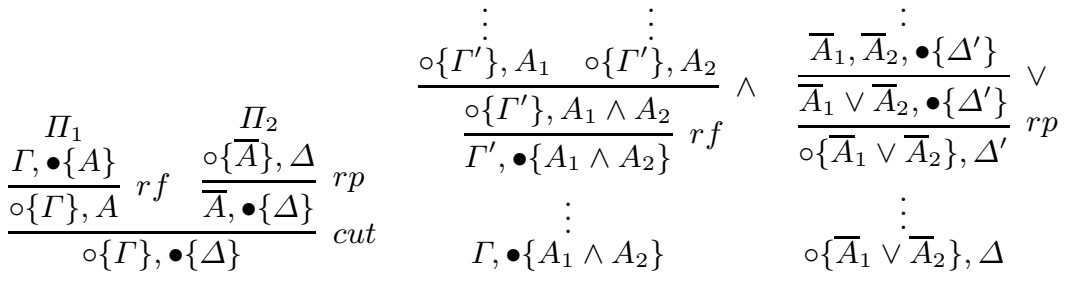

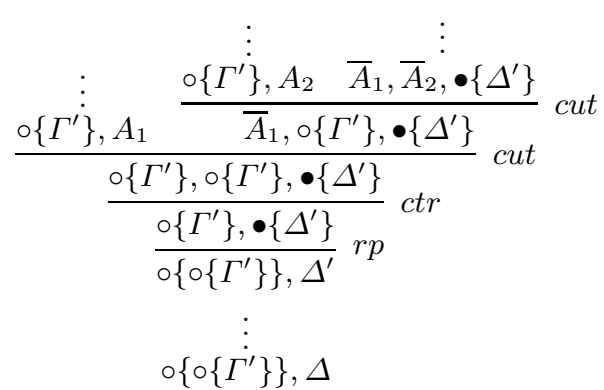

(5)

Fig. 3. Some derivations in SKt.

Cut elimination The main difficulty in proving cut elimination for SKt is in finding the right cut reduction for some cases involving the rules $r p$ and $r f$. For instance, consider the derivation (1) in Figure 3. It is not obvious that there is a cut reduction strategy that works locally without generalizing the cut rule to, e.g., one which allows cut on any sub-sequent in a sequent. Instead, we shall follow a global cut reduction strategy similar to that used in cut elimination for display logics. The idea is that, instead of permuting the cut rule locally, we trace the cut formula $A$ (in $\Pi_{1}$ ) and $\bar{A}$ (in $\Pi_{2}$ ), until they both become principal in their respective proofs, and then apply the cut rule(s) at that point on smaller formulae. Schematically, our simple strategy can be illustrated as follows: Suppose that $\Pi_{1}$ and $\Pi_{2}$ are, respectively, derivation (2) and (3) in Figure 3, that $A=A_{1} \wedge A_{2}$ and there is a single instance in each proof where the cut formula is used. To reduce the cut on $A$, we first transform $\Pi_{1}$ by uniformly substituting $\bullet\{\Delta\}$ for $A$ in $\Pi_{1}$ (see derivation (4) in Figure 3). We then prove the open leaf $\left\{\circ\left\{\circ\left\{\Gamma^{\prime}\right\}\right\}, \Delta\right\}$ by uniformly substituting $\circ\left\{\Gamma^{\prime}\right\}$ for $A$ in $\Pi_{2}$ (see derivation (5) in Figure 3 ). Notice that the cuts on $A_{1}$ and $A_{2}$ introduced in the proof above are on smaller formulae than $A$.

The above simplified explanation implicitly assumes that a uniform substitution of a formula (or formulae) in a proof results in a well-formed proof, and that the cut formulae are not contracted. The precise statement of the proof substitution idea becomes more involved once these aspects are taken into ac- 
count. The formal statement is given in the lemma below. We use the notation $\vdash_{S} \Gamma$ to denote that the sequent $\Gamma$ is provable in the proof system $S$. We write $\vdash_{S} \Pi: \Gamma$ when we want to be explicit about the particular proof $\Pi$ of $\Gamma$. The cut rank of an instance of cut is defined as usual, as the size of the cut formula. The cut rank of a proof $\Pi$, denoted with $\operatorname{cr}(\Pi)$, is the largest cut rank of the cut instances in $\Pi$ (or zero, if there are no cuts in $\Pi$ ). Given a formula $A$, we denote with $|A|$ its size. Given a proof $\Pi$, we denote with $|\Pi|$ its height, i.e., the length of a longest branch in the proof tree of $\Pi$.

Lemma 1. Let $A$ be a non-atomic formula. Suppose $\vdash_{\mathbf{S K t}} \Pi_{1}: \Delta, \bar{A}$ and $\vdash_{\mathbf{S K t}}$ $\Pi_{2}: \Sigma^{k}[A]$, for some $k \geq 1$, and the cut ranks of $\Pi_{1}$ and $\Pi_{2}$ are smaller than $|A|$. Then there exists a proof $\Pi$ such that $\vdash_{\mathbf{S K t}} \Pi: \Sigma^{k}[\Delta]$ and $\operatorname{cr}(\Pi)<|A|$.

Theorem 2. Cut elimination holds for SKt.

\section{System DKt: a contraction-free deep-sequent calculus}

We now consider another sequent system which uses deep inference, where rules can be applied directly to any node within a nested sequent. We call this system DKt, and give its inference rules in Figure 4. Note that there are no structural rules in DKt, and the contraction rule is absorbed into the logical rules. Notice that, reading the logical rules bottom up, we keep the principal formulae in the premise. This is actually not neccessary for some rules (e.g., $\mathbf{\square}, \wedge$, etc.), but this form of rule allows for a better accounting of formulae in our saturation-based proof search procedure (see Section 6).

The following intuitive observation about DKt rules will be useful later: Rules in DKt are characterized by propagations of formulae across different nodes in a nested sequent tree. The shape of the tree is not affected by these propagations, and the only change that can occur to the tree is the creation of new nodes (via the introduction rules $\mathbf{\square}$ and $\square$ ).

System DKt corresponds to Kashima's $S 2 K t$ [12], but with the contraction rule absorbed into the logical rules. Kashima shows that DKt proofs can be encoded into SKt, essentially due to the display property of SKt (Proposition 1) which allows displaying and undisplaying of any node within a nested sequent. Kashima also shows that DKt is complete for tense logic, via semantic arguments. We prove a stronger result: every cut-free $\mathbf{S K}$-proof can be transformed into a DKt-proof, hence DKt is complete and cut is admissible in DKt.

To translate cut-free SKt-proofs into DKt-proofs, we show that all structural rules of SKt are height-preserving admissible in DKt, as stated next.

Lemma 2 (Admissibility of weakening). Suppose $\vdash_{\mathrm{DKt}} \Pi: \Sigma[\Gamma]$. Then for every $\Delta$, there exists $\Pi^{\prime}$ such that $\vdash_{\mathbf{D K t}} \Pi^{\prime}: \Sigma[\Gamma, \Delta]$ and $\left|\Pi^{\prime}\right| \leq|\Pi|$.

The proofs for the following lemmas that concern structural rules that change the shape of the tree of a nested sequent share similarities. That is, the only interesting cases in the proofs are those that concern propagation of formulae across different nodes in a nested sequent. We show here an interesting case in the proof for the admissibility of display postulates. 


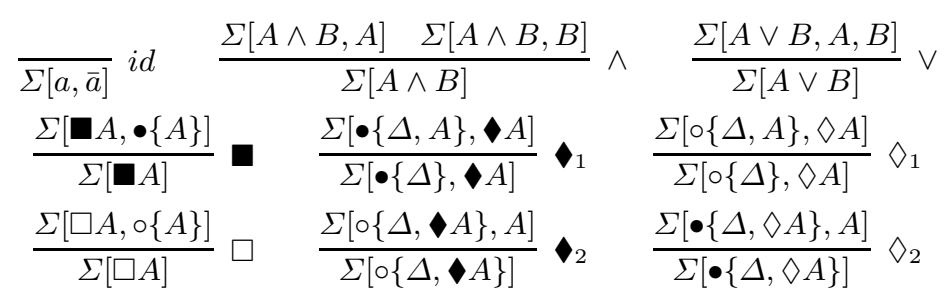

Fig. 4. A contraction-free deep-sequent system

Lemma 3 (Admissibility of display postulates). If $\vdash_{\mathrm{DKt}} \Pi: \Gamma, \bullet\{\Delta\}$ then there exists $\Pi^{\prime}$ such that $\vdash_{\mathbf{D K t}} \Pi^{\prime}: \circ\{\Gamma\}, \Delta$ and $\left|\Pi^{\prime}\right| \leq|\Pi|$.

Proof. By induction on $|\Pi|$. The non-trivial cases are when there is an exchange of formulae between $\Gamma$ and $\Delta$. One example is when $\Pi$ is as shown below left. Then $\Pi^{\prime}$ is as shown below right where $\Pi_{1}^{\prime}$ is obtained from induction hypothesis:

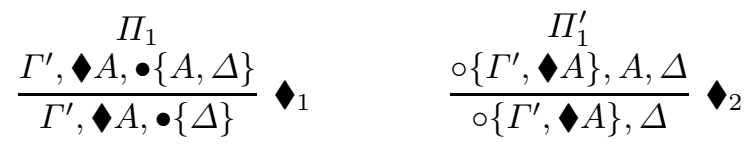

Lemma 4 (Admissibility of display postulates). If $\vdash_{\mathrm{DKt}} \Pi: \Gamma, \circ\{\Delta\}$ then there exists $\Pi^{\prime}$ such that $\vdash_{\mathbf{D K t}} \Pi^{\prime}: \bullet\{\Gamma\}, \Delta$ such that $\left|\Pi^{\prime}\right| \leq|\Pi|$.

Lemma 5 (Admissibility of contraction). If $\vdash_{\text {DKt }} \Pi: \Sigma[\Delta, \Delta]$ then there exists $\Pi^{\prime}$ such that $\vdash_{\mathrm{DKt}} \Pi^{\prime}: \Sigma[\Delta]$ and $\left|\Pi^{\prime}\right| \leq|\Pi|$.

Theorem 3. For every sequent $\Gamma, \vdash_{\mathbf{S K t}} \Gamma$ if and only if $\vdash_{\mathbf{D K t}} \Gamma$.

A consequence of Theorem 3 is that the general contraction rule in SKt can be replaced by formula contraction. This can be proved as follows: take a cut-free proof in SKt, translate it to $\mathbf{D K t}$ and then translate it back to SKt. Since general contraction is admissible in $\mathbf{D K t}$, and since the translation from DKt to SKt does not use general contraction (only formula contraction), we can effectively replace the general contraction in SKt with formula contraction.

An interesting feature of DKt is that in a proof of a sequent, the 'color' of a (formula or structural) connective does not change when moving from premise to conclusion or vice versa. Let us call a formula (a sequent, a rule) purely modal if it contains no black connectives. It is easy to see that if a purely modal formula (sequent) is provable in $\mathbf{D K t}$, then it is provable using only purely modal rules. Let $\mathbf{D K}=\left\{i d, \wedge, \vee, \square, \diamond_{1}\right\}$, i.e., it is the set of purely modal rules of DKt. The above observation leads to the following "separation" result:

Theorem 4. For every modal formula $\varphi, \vdash_{\mathbf{D K}} \varphi$ iff $\varphi$ is a theorem of $K$.

This completeness result for $\mathbf{D K}$ is known from [3]; what we show here is how it can be derived as a consequence of completeness of DKt. 


$$
\begin{array}{lll}
\frac{\Sigma[\diamond A, A]}{\Sigma[\diamond A]} T_{a} & \frac{\Sigma[\diamond A, \bullet\{\diamond A, \Delta\}]}{\Sigma[\diamond A, \bullet\{\Delta\}]} 4_{a} & \frac{\Sigma[\diamond A, \circ\{\diamond A, \Delta\}]}{\Sigma[\diamond A, \circ\{\Delta\}]} 4_{c} \\
\frac{\Sigma[\diamond A, A]}{\Sigma[\diamond A]} T_{b} & \frac{\Sigma[\circ\{\Delta, \diamond A\}, \diamond A]}{\Sigma[\circ\{\Delta, \diamond A\}]} 4_{b} & \frac{\Sigma[\bullet\{\Delta, \diamond A\}, \diamond A]}{\Sigma[\bullet\{\Delta, \diamond A\}]} 4_{d}
\end{array}
$$

Fig. 5. Additional propagation rules for DS4

\section{Proof systems for some extensions of tense logic}

We now consider extensions of tense logic with some modal axioms. We show that, for each extension, there is a shallow system that modularly extends SKt for which cut elimination holds. By modular extension we mean that the rules of the extended systems are the rules of SKt plus some structural rules that are derived directly from the modal axioms. We then show that for each extension, there is also a corresponding deep-inference system which is equivalent to the shallow one. Again, as with DKt, the rules for the deep system are characterized by propagations of formulae across different nodes in the nested sequents. However, the design of the rules for the deep system is not as modular as its shallow counterpart, since it needs to take into account the closure of the axioms.

Cut elimination holds for all the extensions discussed in the following. Their proofs are omitted as they are a straightforward adaptation of the cut elimination proof for SKt. This is because the proof substitution technique used for cut elimination in SKt relies on rule applications being invariant under formula substitution. More precisely, all the additional structural rules that we shall consider have the following property: If there is an instance of a structural rule $\rho$ (below left) then instantiating the occurrences of $A$ in the multi-context $\Sigma_{1}$ and $\Sigma_{2}$ with any structure $\Delta$ yields a valid instance of $\rho$ (below right):

$$
\frac{\Sigma_{2}^{k}[A]}{\Sigma_{1}^{k}[A]} \rho \quad \frac{\Sigma_{2}^{k}[\Delta]}{\sum_{1}^{k}[\Delta]} \rho .
$$

Hence the proof substitution technique for cut elimination goes through essentially unchanged for the extended logic. This property of the structural rules is similar to Belnap's condition (C6) for cut elimination for display logics [2].

A primitive axiom is an axiom of the form $A \rightarrow B$ where both $A$ and $B$ are built using propositional variables, $\wedge, \vee, \diamond$, and $\diamond$. Kracht [13] shows that any extension of tense logic with primitive axioms has a display calculus which enjoys cut elimination. He shows that any such axiom can be turned into a left structural rule. The axioms we consider next are contrapositives of primitive axioms, so Kracht's translation from axioms to structural rules in our formalism gives right structural rules. We illustrate here a few cases of primitive axioms for which one can also get corresponding deep sequent systems.

Modal tense logic S4 Consider an extension of SKt with the following axioms:

$$
T: \square A \rightarrow A \quad \square A \rightarrow A \quad 4: \square A \rightarrow \square \square A \quad \mathbf{\square} A \rightarrow \mathbf{\square} A .
$$




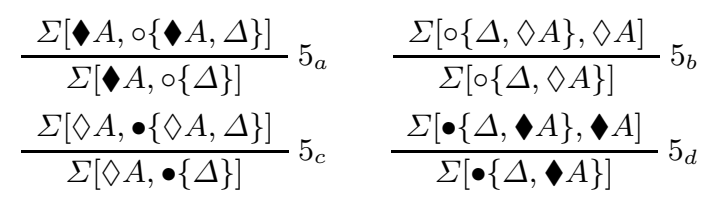

Fig. 6. Additional propagation rules for DS5

These axioms translate into the following structural rules, whose soundness is immediately derivable from the axioms:

$$
\frac{\Gamma, \bullet\{\Delta\}}{\Gamma, \Delta} T_{p} \quad \frac{\Gamma, \circ\{\Delta\}}{\Gamma, \Delta} T_{f} \quad \frac{\Gamma, \bullet\{\Delta\}}{\Gamma, \bullet\{\bullet\{\Delta\}\}} 4_{p} \quad \frac{\Gamma, \circ\{\Delta\}}{\Gamma, \circ\{\circ\{\Delta\}\}} 4_{f}
$$

Definition 3 (System SS4). System SS4 is SKt plus $T_{p}, T_{f}, 4_{p}$ and $4_{f}$.

Theorem 5. Cut elimination holds for SS4.

Definition 4 (System DS4). System DS4 is DKt plus the propagation rules given in Figure 5.

Some of the modal rules of DS4 coincide with Brünnler's rules for $T$ and 4 in [3]. The rules of DS4 can be shown to be derivable in SS4.

Lemma 6. Every rule of DS4 is derivable in SS4.

To prove the equivalence of SS4 and DS4, we need to prove the analogs of Lemma $2-5$. These are again a straightforward adaptation of the previous proofs, and are omitted here. Additionally, we need to show that the structural rules for the axioms $T$ and 4 are also admissible in DS4. The principle behind the proofs of admissibility for these structural rules is again the same; the nontrivial cases we need to consider are those that concern propagation of formulae across structures affected by the structural rules.

Theorem 6. For every $\Gamma$, we have $\vdash_{\mathbf{S S}_{4}} \Gamma$ if and only if $\vdash_{\mathbf{D S} 4} \Gamma$.

Modal tense logic S5 We can obtain S5 from SS4 by collapsing $\square$ and That is, the symmetry axiom $B: A \rightarrow \square \diamond A$ splits into two axioms given below, which translate straightforwardly into two structural rules.

$$
B 1: \square A \rightarrow \square A \quad \frac{\Gamma, \bullet\{\Delta\}}{\Gamma, \circ\{\Delta\}} B_{1} \quad B 2: \square A \rightarrow \mathbf{\square} A \quad \frac{\Gamma, \circ\{\Delta\}}{\Gamma, \bullet\{\Delta\}} B_{2}
$$

Definition 5 (System SS5). System SS5 is SS4 plus the rules $B_{1}$ and $B_{2}$.

Theorem 7. Cut elimination holds for SS5.

Definition 6 (System DS5). System DS5 is DS4 plus the propagation rules given in Figure 6. 
Function Prove (Sequent $\Xi$ ) : Bool

1. Let $T=\operatorname{tree}(\Xi)$

2. If the $i d$ rule is applicable to any node in $T$, return True

3. Else if there is some node $\Theta \in T$ that is not saturated

(a) If $A \vee B \in \Theta$ and $A \notin \Theta$ or $B \notin \Theta$ then let $\Xi_{1}$ be the premise of the $\vee$ rule applied to $A \vee B \in \Theta$. Return Prove $\left(\Xi_{1}\right)$.

(b) If $A \wedge B \in \Theta$ and $A \notin \Theta$ and $B \notin \Theta$ then let $\Xi_{1}$ and $\Xi_{2}$ be the premises of the $\wedge$ rule applied to $A \wedge B \in \Theta$. Return True iff Prove $\left(\Xi_{1}\right)=$ True and $\operatorname{Prove}\left(\Xi_{2}\right)=$ True.

4. Else if there is some node $\Theta \in T$ that is not realised, i.e. some $B=\square A$ ( $B=$ is not realised

(a) Let $\Xi_{1}$ be the premise of the $\square$ ( $\left.\boldsymbol{\square}\right)$ rule applied to $B \in \Theta$. Return Prove $\left(\Xi_{1}\right)$.

5. Else if there is some node $\Theta$ that is not propagated

(a) Let $\rho$ be the rule corresponding to the requirement of Definition 9 that is not met, and let $\Xi_{1}$ be the premise of $\rho$. Return Prove $\left(\Xi_{1}\right)$.

6. Else return False

Fig. 7. Proof search strategy for DKt

Lemma 7. Every rule of DS5 is derivable in SS5.

We can prove the analogs of Lemma $2-5$ and admissibility of the rules corresponding to the axioms of SS4 and structural rules $B_{1}$ and $B_{2}$. Note that DS5 captures $S 5=K T 45$ rather than $S 5=K T 4 B$.

Theorem 8. For every $\Gamma$, we have $\vdash_{\mathbf{S S 5}} \Gamma$ if and only if $\vdash_{\mathbf{D S 5}} \Gamma$.

\section{Proof search}

We can devise terminating proof search strategies for our deep sequent calculi. While traditional tableaux methods operate on a single node at a time, our proof search strategies will consider the whole tree. Following Kashima, first we define a mapping from sequents to trees.

A node is a set of formulae. A tree is a node with 0 or more children, where each child is a tree, and each child is labelled as either a o-child, or a $\bullet$-child. Given a sequent $\Xi=\Theta, \circ\left\{\Gamma_{1}\right\}, \cdots, \circ\left\{\Gamma_{n}\right\}, \bullet\left\{\Delta_{1}\right\}, \cdots, \bullet\left\{\Delta_{m}\right\}$, where $\Theta$ is a set of formulae and $n \geq 0$ and $m \geq 0$, the tree $\operatorname{tree}(\Xi)$ represented by $\Xi$ is:

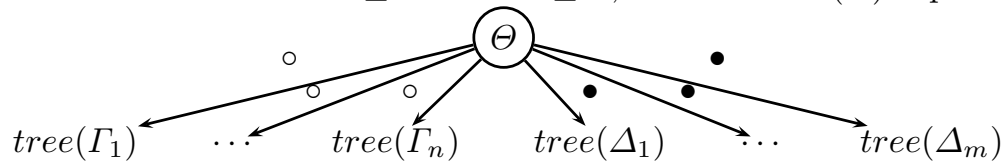

Definition 7. A set of formulae $\Theta$ is saturated iff it satisfies:

1. If $A \vee B \in \Theta$ then $A \in \Theta$ and $B \in \Theta$.

2. If $A \wedge B \in \Theta$ then $A \in \Theta$ or $B \in \Theta$.

Definition 8. Given a tree $T$ and a node $\Theta \in T$, a formula $\square A \in \Theta(\square A \in \Theta)$ is realised iff there exists a $\circ$-child ( $\bullet$-child) $\Gamma$ of $\Theta$ in $T$ with $A \in \Gamma$. 


\subsection{Proof Search in DKt}

Figure 7 gives a proof search strategy for DKt. The application of a rule deep inside a sequent can be viewed as focusing on a particular node of the tree. The rules of DKt can then be viewed as operations on the tree encoded in the sequent. In particular, Step 3 saturates a node locally, Step 4 appends new nodes to the tree, and Step 5 moves $\diamond(\checkmark)$ prefixed formulae between neighbouring nodes.

Definition 9. Given a tree $T$ and a node $\Theta \in T$, we say $\Theta$ is propagated iff:

$\diamond_{1}$ : for every $\diamond A \in \Theta$ and for every o-child $\Gamma$ of $\Theta$, we have $A \in \Gamma$

${ }_{1}$ : for every $A \in \Theta$ and for every $\bullet$-child $\Gamma$ of $\Theta$, we have $A \in \Gamma$

$\diamond_{2}$ : for every $\bullet$-child $\Gamma$ of $\Theta$ and for every $\diamond A \in \Gamma$, we have $A \in \Theta$

${ }_{2}$ : for every o-child $\Gamma$ of $\Theta$ and for every $A \in \Gamma$, we have $A \in \Theta$

The degree of a formula is the maximum number of nested modalities:

$$
\begin{aligned}
\operatorname{deg}(p) & =0 \\
\operatorname{deg}(A \# B) & =\max (\operatorname{deg}(A), \operatorname{deg}(B)) \text { for } \# \in\{\wedge, \vee\} \\
\operatorname{deg}(\# A) & =1+\operatorname{deg}(A) \text { for } \# \in\{\square, \diamond, \mathbf{\square}, \bullet\} .
\end{aligned}
$$

The degree of a set of formulae is the maximum degree over all its members. We write $s f(A)$ for the subformulae of $A$, and define the set of subformulae of a set $\Theta$ as $s f(\Theta)=\bigcup_{A \in \Theta} s f(A)$. For a sequent $\Xi$ we define $s f(\Xi)$ as below:

$$
\begin{aligned}
& \Xi \quad=\Theta, \circ\left\{\Gamma_{1}\right\}, \cdots, \circ\left\{\Gamma_{n}\right\}, \bullet\left\{\Delta_{1}\right\}, \cdots, \bullet\left\{\Delta_{m}\right\} \\
& s f(\Xi)=\operatorname{sf}(\Theta) \cup s f\left(\Gamma_{1}\right) \cup \cdots \cup s f\left(\Gamma_{n}\right) \cup s f\left(\Delta_{1}\right) \cup \cdots \cup s f\left(\Delta_{m}\right) .
\end{aligned}
$$

Theorem 9. Function Prove terminates for any input sequent $\Xi$.

\subsection{Proof Search in DS4}

Let $\mathbf{D S}^{-}{ }^{-}$denote the system DS4 minus the rules $\diamond_{1}, \diamond_{2}, \diamond_{1}, \diamond_{2}$.

Theorem 10. For every $\Gamma$, we have $\vdash_{\mathbf{D S}^{-}} \Gamma$ if and only if $\vdash_{\mathbf{D S} 4} \Gamma$.

Proof. $\Rightarrow$ : obvious since every rule of $\mathbf{D S}^{-}$is a rule of DS4. $\Leftarrow$ : by induction on the height of the proof of $\vdash_{\mathbf{D S} 4} \Gamma$, using the admissibility of $\diamond_{1}, \diamond_{2}, \diamond_{1}, \diamond_{2}$.

We now modify the Prove function for proof search in DS4. The saturation and propagatation of $\diamond$ - and $\downarrow$-prefixed formulae need to cater for reflexivity and transitivity respectively. Moreover, a loop check and blocking on the creation of new nodes is required, since a naive approach leads to non-termination [9]. We implement the loop check by adding histories to our nodes (in our case in the form of tagged formulae), thus extending Heuerding's approach [9] to tense logic.

A tagged formula is a formula of the form $A^{*}$. For $\# \in\{\square, \diamond, \boldsymbol{\square}, \downarrow$, we write $(\# \Gamma)^{*}$ to mean a set of tagged \#-formulae. In the following, the nodes in our trees will consist of sets of formulae and tagged formulae. Note that we use tagged formulae for book-keeping only; tagged formulae are never principal in inference rule applications. Let $\mathbf{D S} \mathbf{4}^{*}$ be $\mathbf{D S} 4^{-}$with the $T_{a}, T_{b}, \square, \boldsymbol{\square}$ rules replaced by the following. For simplicity, we write them directly as tree expansion rules: 
$T_{a}^{1}\left(T_{b}^{1}\right)$ : If some node $\Theta$ contains an untagged $\diamond A(\diamond A)$, add $A$ to $\Theta$, tag $\diamond A$ $(\diamond A)$ and untag all $\square(\boldsymbol{\square})$ formulae.

$T_{a}^{2}\left(T_{b}^{2}\right)$ : If some $\Theta$ contains a tagged $A(\diamond A)$, add $A$ to $\Theta$.

$\square A$ : If some node $\Theta$ contains an unrealised, untagged $\square A$, create an o-child $\left\{(\square \Gamma)^{*},(\diamond \Delta)^{*}, A\right\}$, where $\square \Gamma$ are all the $\square$ formulae in $\Theta$, and $(\diamond \Delta)^{*}$ are all the tagged $\diamond$ formulae in $\Theta$.

$\mathbf{\square} A$ : If some node $\Theta$ contains an unrealised, untagged $\boldsymbol{\square} A$, create a $\bullet$-child $\left\{(\boldsymbol{\square} \Gamma)^{*},(\checkmark)^{*}, A\right\}$, where $\Gamma$ are all the $\mathbf{\square}$ formulae in $\Theta$, and $(\checkmark)^{*}$ are all the tagged formulae in $\Theta$.

The intuition of tagging is that $\square$ ( $\square$ ) formulae are only expanded once within each cycle of repeated $\diamond(\diamond)$ formulae. If an untagged $\diamond(\diamond)$ formula is encountered, rule $T_{a}^{1}\left(T_{b}^{1}\right)$ removes the tags from all tagged $\square(\boldsymbol{\square})$ formulae so that they can be expanded again. Eventually all $\diamond(\downarrow)$ formulae will be tagged, so the $\square$ (ם) formulae will also remain tagged and the $\square$ (ם) rules will be blocked.

Definition 10. A set of formulae $\Theta$ is $S 4$-saturated iff it is saturated and $\diamond A \in$ $\Theta$ or $A \in \Theta$ implies $A \in \Theta$.

Definition 11. A node $\Theta$ in a given tree $T$ is $S 4$-propagated iff:

$4_{a}$ : for every $A \in \Theta$ and for every $\bullet$-child $\Gamma$ of $\Theta$, we have $A \in \Gamma$

$4_{b}$ : for every $\circ$-child $\Gamma$ of $\Theta$ and for every $A \in \Gamma$, we have $A \in \Theta$

$4_{c}$ : for every $\diamond A \in \Theta$ and for every o-child $\Gamma$ of $\Theta$, we have $\diamond A \in \Gamma$

$4_{d}$ : for every $\bullet$-child $\Gamma$ of $\Theta$ and for every $\diamond A \in \Gamma$, we have $\diamond A \in \Theta$

Let ProveS4 be the function Prove from Figure 7, modified as follows:

1. Replace "saturated" with "S4-saturated" and add four sub-steps to Step 3 for $\diamond$ - and $\checkmark$-formulae based on the rules $T_{a}^{1}, T_{b}^{1}, T_{a}^{2}$ and $T_{b}^{2}$.

2. Replace "propagated" with "S4-propagated" in Step 5, and use Definition 11 instead of Definition 9.

Lemma 8. For every $\mathbf{D S} \mathbf{4}^{*}$-derivation $\Pi$, for every sequent $\Xi \in \Pi$, the maximum number of consecutive o-edges in tree $(\Xi)$ is $m^{2}$, where $m=|s f(\Xi)|$.

Lemma 9. For every $\mathbf{D S} \mathbf{4}^{*}$-derivation $\Pi$, for every sequent $\Xi \in \Pi$, the maximum number of consecutive $\bullet$-edges in tree $(\Xi)$ is $m^{2}$, where $m=|s f(\Xi)|$.

Theorem 11. Function ProveS4 terminates for any input sequent $\Xi$.

Proof. Let $T=\operatorname{tree}(\Xi)$. The argument for Steps 3 and Step 5 is similar for the proof of Theorem 9. We need show that the depth of $\mathrm{T}$ is bounded by the loop check side conditions on the rules $T_{a}^{1}, T_{b}^{1}$,

For a contradiction, suppose there exists a $T$ of infinite depth, i.e., $T$ contains an infinite branch. By Lemmas 8 and 9, an infinite branch must contain an infinite number of alternations between sequences of o-labelled edges and •labelled edges. Since o-children are created by applications of the $\square$-rule and -children are created by applications of the $\mathbf{\square}$-rule, there must be an infinite 
number of alternating $\square$ and rule applications (with any other rule applications in between). However, every such alternation decreases the degree of the node by at least 1 , since the $\square$ (ם) rule removes the outer $\square$ (ם) from the principal formula, and $\diamond(\diamond)$ formulae can only be propagated across $\circ(\bullet)$ edges. Thus an infinite number of alternating $\square$ and $\boldsymbol{\square}$ rules is impossible. Contradiction.

\section{Related Work and Future Work}

Bernardi [1] appears to be the first to have noticed the connection between deep inference and residuation in display logic in the context of categorial grammar, although they do not give an explicit proof of this correspondence. Brünnler [3, 4] and Poggiolesi [15] have given deep inference calculi for the modal logic $\mathrm{K}$ and some extensions. Brünnler has recently shown that the deep-inference-based cut-elimination technique for $\mathrm{K}$ [3] can be extended to prove cut elimination for Kashima's deep inference calculus for Kt. ${ }^{1}$ In his proof, a crucial step is a proof of the admissibility of a "deep" version of residuation:

$$
\frac{\Sigma[\bullet\{\circ\{\Delta\}, \Gamma\}]}{\Sigma[\Delta, \bullet\{\Gamma\}]} \quad \frac{\Sigma[\circ\{\bullet\{\Delta\}, \Gamma\}]}{\Sigma[\Delta, \circ\{\Gamma\}]}
$$

It will be interesting to compare the direct proof of cut elimination in deep systems (without residuation) to the one in shallow system (with residuation).

Indrzejczak [11] and Trzesicki [16] have given cut-free sequent-like calculi for tense logic. In each such calculus there is a rule (or rules) which allow us to "return" to previously seen worlds when the rules are viewed from the perspective of counter-model construction. However, Trzesicki's calculus has a large degree of non-determinism and is therefore not suitable for proof search. In contrast, our system DKt and its extension to tense S4 admits a simple proof search strategy and termination argument. Indrzejczak's calculus is suitable for proof search but lacks a natural notion of a cut rule and cut-elimination. It is also possible to give proof calculi for many modal and tense logics using semantic methods such as labelled deduction [14] and graph calculi [5], but we prefer purely syntactic methods since they can potentially be applied to logics with more complicated semantics such as substructural logics.

The description logic community have already built extremely efficient theorem provers for Kt.S4 in its incarnation as ALCI with transitive roles [10], so our terminating calculus for Kt.S4 is not very exciting. However, Horrocks et. al. do not consider proof-theoretic issues such as cut-elimination.

It remains to be seen whether we can extend our results to the primitive extensions of modal tense logic in a systematic way, and also whether deep inference can be used to tame other display calculi with more complex binary residuation principles like those in substructural logics [1]. Another interesting direction is the addition of (first-order) quantifiers. An approach to this would be to consider quantifiers as modal operators, with appropriate display postulates,

\footnotetext{
${ }^{1}$ K. Brünnler. Personal communication.
} 
such as the ones developed in [18]. A simple Haskell implementation of DKt is available at: http://users.rsise.anu.edu.au/ linda/DKt.html.

Acknowledgment: We thank the anonymous referees for their helpful comments on an earlier draft of the paper.

\section{References}

1. C. Areces and R. Bernardi. Analyzing the core of categorial grammar. Journal of Logic, Language, and Information, 13(2):121-137, 2004.

2. N. Belnap. Display logic. Journal of Philosophical Logic, 11:375-417, 1982.

3. K. Brünnler. Deep sequent systems for modal logic. In G. G. et al, editor, Advances in Modal Logic 6, pages 107-119. College Publications, 2006.

4. K. Brünnler. Deep sequents for modal logic. Unpublished, 2007.

5. M. A. Castilho, L. F. D. Cerro, O. Gasquet, and A. Herzig. Modal tableaux with propagation rules and structural rules. Fundamenta Informaticae, 32(3/4):281297, 1997.

6. R. Goré. Gaggles, Gentzen and Galois: How to display your favourite substructural logic. Logic Journal of the IGPL, 6(5):669-694, 1998.

7. R. Goré. Substructural logics on display. LJIGPL, 6(3):451-504, 1998.

8. R. Goré, L. Postniece, and A. Tiu. Cut-elimination and proof-search for biintuitionistic logic using nested sequents. In in AiML. College Publications, 2008.

9. A. Heuerding, M. Seyfried, and H. Zimmermann. Efficient loop-check for backward proof search in some non-classical propositional logics. In Analytic Tableaux and Related Methods, volume 1071 of LNAI, pages 210-225, 1996.

10. I. Horrocks, U. Sattler, and S. Tobies. Practical reasoning for very expressive description logics. Logic Journal of the IGPL, 8(3):239-264, 2000.

11. A. Indrzejczak. Multiple sequent calculus for tense logics. International Conference on Temporal Logic, Leipzig 2000. 93-104.

12. R. Kashima. Cut-free sequent calculi for some tense logics. Studia Logica, 53:119 135, 1994.

13. M. Kracht. Power and weakness of the modal display calculus. In H. Wansing, editor, Proof Theory of Modal Logics, pages 92-121. Kluwer, 1996.

14. S. Negri. Proof analysis in modal logic. JPL, 34(5-6):507-544, 2005.

15. F. Poggiolesi. The tree-hypersequent method for modal propositional logic. Trends in Logic: Towards Mathematical Philsophy, pages 9-30, 2009.

16. K. Trzesicki. Gentzen-style axiomatization of tense logic. Bulleting of the Section of Logic, 13(2):75-84, 1984.

17. H. Wansing. Sequent calculi for normal modal proposisional logics. Journal of Logic and Computation, 4(2):125-142, Apr. 1994.

18. H. Wansing. Displaying Modal Logic. Kluwer Academic Publishers, 1998. 


\section{A Proofs for Section 3}

Lemma 10. Let $\Sigma_{1}[X], \Sigma_{2}[Y]$ be a sequent with a unique occurrence of a formula $X$ and a unique occurrence of a formula $Y$ ( $X$ and $Y$ are distinct formulae). Then there exists a context $\Sigma[]$ such that $\Sigma_{1}[X], \Sigma_{2}[Y]$ is derivable from $X, \Sigma[Y]$ using only the rules rp and $r f$.

Proof. By induction on the size of the context $\Sigma_{1}[]$. The non-trivial cases are when $\Sigma_{1}[]$ is of the form $\circ\left\{\Sigma_{3}[]\right\}$ or $\bullet\left\{\Sigma_{3}[]\right\}$. We consider the former case here; the latter can be handled analogously. By induction hypothesis, there exists $\Sigma^{\prime}[]$ such that $\Sigma_{3}[X], \bullet\left\{\Sigma_{2}[Y]\right\}$ is derivable from $X, \Sigma^{\prime}[Y]$. Let $\Sigma[]=\Sigma^{\prime}[]$. Then we have a derivation

$$
\begin{gathered}
X, \Sigma^{\prime}[Y] \\
\vdots \\
\frac{\Sigma_{3}[X], \bullet\left\{\Sigma_{2}[Y]\right\}}{\circ\left\{\Sigma_{3}[X]\right\}, \Sigma_{2}[Y]} r p
\end{gathered}
$$

that uses only $r p$ and $r f$.

Proposition 1. Let $\Sigma[\Delta]$ be a sequent. Then there exists a sequent $\Gamma$ such that $\Sigma[\Delta]$ is derivable from $\Delta, \Gamma$ and vice versa, using only the rules rp and $r f$.

Proof. We first show the forward direction, i.e., deriving $\Sigma[\Delta]$ from $\Gamma, \Delta$. Applying Lemma 10 with $\Sigma_{1}[]=\Sigma[]$ and $\Sigma_{2}[]=[]$, we have context $\Sigma^{\prime}[]$ such that there exists a derivation $\Pi$ from $X, \Sigma^{\prime}[Y]$ to $\Sigma[X], Y$, using only $r p$ and $r f$. Let $\Gamma=\Sigma^{\prime}[\{\}]$. Now, by instantiating $X$ to $\Delta$ and $Y$ to \{\} in $\Pi$, we get a derivation of $\Sigma[\Delta]$ from $\Delta, \Gamma$ using only $r p$ and $r f$.

For the converse, we first observe that the rules $r p$ and $r f$ are dual to each other. That is, if we turn the rule $r p$ upside down, we get $r f$. Therefore, to get a derivation of $\Delta, \Gamma$ from $\Sigma[\Delta]$, we just turn the derivation obtained in the previous case upside down, and rename the rule $r p$ to $r f$ and vice versa.

\section{A.1 Soundness and completeness}

Theorem 1 is a simple corollary of the following lemmas.

Lemma 11 (Soundness). Every SKt-derivable Kt formula is valid.

Proof. We show that for every rule $\rho$ of $\mathbf{S K t}$

$$
\frac{\Gamma_{1} \quad \cdots \quad \Gamma_{n}}{\Gamma} \rho
$$

the following holds: if for every $i \in\{1, \ldots, n\}$, the formula $\tau\left(\Gamma_{i}\right)$ is valid then the formula $\tau(\Gamma)$ is valid. Note that since the exchange rule is implicit in our sequent system, the following soundness argument has to be read modulo associativity and commutativity of the disjunction connective (which is the interpretation of the sequent's comma). 
Since the formula-translation $\tau(\Gamma) \vee a \vee \bar{a}$ of the $i d$ rule is obviously valid, it then follows that every formula derivable in SKt is also valid. We give the formula translations for some of the structural rules and modal rules, the others are similar or easier:

$r f:$ if $\tau(\Gamma) \vee \square(\tau(\Delta))$ valid then $\mathbf{\square}(\tau(\Gamma)) \vee \tau(\Delta)$ valid rp: if $\tau(\Gamma) \vee \mathbf{\square}(\tau(\Delta))$ valid then $\square(\tau(\Gamma)) \vee \tau(\Delta)$ valid $\square$ : if $\tau(\Gamma) \vee \square A$ valid then $\tau(\Gamma) \vee \square A$ valid $\diamond:$ if $\tau(\Gamma) \vee \square(\tau(\Delta) \vee A)$ valid then $\tau(\Gamma) \vee \square(\tau(\Delta)) \vee \diamond A$ valid

Lemma 12 (Completeness). Every Kt-theorem is SKt-derivable.

Proof. The following are derivations of Axioms 1 and 3, the other axioms are similar. Double lines abbreviate derivations:

\begin{tabular}{|c|c|c|}
\hline$\circ\{\}, \bar{A}, A$ & $A \wedge \bar{B}, A, \bar{A}, B, \bullet\{\}$ & $A \wedge \bar{B}, \bar{B}, \bar{A}, B, \bullet\{\}$ \\
\hline$\bullet\{\bar{A}, A\}$ & \multicolumn{2}{|c|}{$A \wedge \bar{B}, \bar{A}, B, \bullet\{\}$} \\
\hline$\bullet\{\bar{A}\}, \diamond A$ & \multicolumn{2}{|c|}{$\circ\{A \wedge \bar{B}, \bar{A}, B\}$} \\
\hline $\bar{A}, \circ\{\diamond A\}$ & \multicolumn{2}{|c|}{$\diamond(A \wedge \bar{B}), \diamond \bar{A}, \circ\{B\}$} \\
\hline $\bar{A}, \square \diamond A$ & \multicolumn{2}{|c|}{$\diamond(A \wedge \bar{B}), \diamond \bar{A}, \square B$} \\
\hline $\bar{A} \vee \square \backslash A$ & \multicolumn{2}{|c|}{$\overline{\bar{\nabla}(A \wedge \bar{B}) \vee \diamond \bar{A} \vee \square B}$} \\
\hline
\end{tabular}

The following are derivations of the rules $M P, N e c \square$ and $N e c$

$$
\frac{\bar{A} \vee B}{\frac{\frac{A}{A \wedge \bar{B}, A, B} w k \frac{}{A \wedge \bar{B}, \bar{B}, B}}{A \wedge \bar{B}, B} \text { cut }} \wedge
$$

$\frac{\frac{A}{A, \bullet\{\}} w k}{\frac{\circ\{A\}}{\square A} \square} \quad \frac{\frac{A}{A, \circ\{\}} w k}{\frac{\bullet\{A\}}{\square}} r f$

\section{A.2 Cut elimination}

Lemma 13. If $\vdash_{\mathbf{S K t}} \Pi_{1}: \Gamma$, a and $\vdash_{\mathbf{S K t}} \Pi_{2}: \Sigma^{k}[\bar{a}]$, where $k \geq 1$ and both $\Pi_{1}$ and $\Pi_{2}$ are cut free, then there exists a cut free $\Pi$ such that $\vdash_{\mathbf{S K t}} \Pi: \Sigma^{k}[\Delta]$.

Proof. By induction on $\left|\Pi_{2}\right|$. For the base cases, the non-trivial case is when $\Pi_{2}$ ends with $i d$ and $\bar{a}$ is active in the rule, i.e., $\Sigma^{k}[\bar{a}]=\Sigma_{1}^{k-1}[\bar{a}], \bar{a}, a$ and $\Pi_{2}$ is

$$
\overline{\Sigma^{k}[\bar{a}]=\Sigma_{1}^{k-1}[\bar{a}], \bar{a}, a} i d
$$


Then we construct $\Pi$ follows:

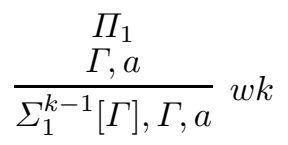

The inductive cases follow straightforward from the induction hypothesis.

Lemma 14. Suppose $\vdash_{\mathbf{S K t}} \Pi_{1}: \Delta, A, \vdash_{\mathbf{S K t}} \Pi_{2}: \Delta, B, \vdash_{\mathbf{S K t}} \Pi: \Sigma^{k}[\bar{A} \vee \bar{B}]$, for some $k \geq 1$, and the cut ranks of $\Pi_{1}, \Pi_{2}$ and $\Pi$ are smaller than $|A \wedge B|$. Then there exists a proof $\Pi^{\prime}$ such that $\vdash_{\mathbf{S K t}} \Pi^{\prime}: \Sigma^{k}[\Delta]$ and $\operatorname{cr}(\Pi)<|A \wedge B|$.

Proof. By induction on $|\Pi|$. Most cases are straightforward. The only non-trivial case is when $\bar{A} \vee \bar{B}$ is principal in the last rule of $\Pi$, i.e., $\Pi$ is of the form

$$
\frac{\Sigma_{1}^{k-1}[\bar{A} \vee \bar{B}], \bar{A} \vee \bar{B}}{\sum_{1}^{k-1}[\bar{A} \vee \bar{B}], \bar{A} \vee \bar{B}}
$$

By induction hypothesis, we have a cut-free proof $\Psi^{\prime}$ such that

$$
\vdash_{\mathbf{S K t}} \Psi^{\prime}: \Sigma_{1}^{k-1}[\Delta], \bar{A}, \bar{B}
$$

The proof $\Pi^{\prime}$ is constructed as follows:

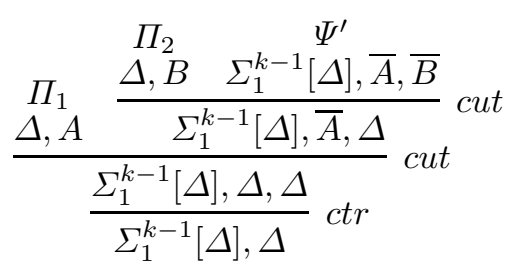

Lemma 15. Suppose $\vdash_{\mathbf{S K t}} \Pi_{1}: \Delta, A, B$ and $\vdash_{\mathbf{S K t}} \Pi_{2}: \Sigma^{k}[\bar{A} \wedge \bar{B}]$, for some $k \geq 1$, and the cut ranks of $\Pi_{1}$ and $\Pi_{2}$ are smaller than $|A \vee B|$. Then there exists a proof $\Pi$ such that $\vdash_{\mathbf{S K t}} \Pi: \Sigma^{k}[\Delta]$ and $\operatorname{cr}(\Pi)<|A \vee B|$.

Proof. This is proved analogously to Lemma 14.

To prove the next two lemmas, we use the following derived rules:

$$
\frac{\Gamma, \circ\{\Delta\}_{1}, \circ\{\Delta\}_{2}}{\Gamma, \circ\left\{\Delta_{1}, \Delta_{2}\right\}} d 1 \quad \frac{\Gamma, \bullet\{\Delta\}_{1}, \bullet\{\Delta\}_{2}}{\Gamma, \bullet\left\{\Delta_{1}, \Delta_{2}\right\}} d 2
$$


These two rules are derivable using $r p, r f$, ctr and $w k$. The rule $d 1$ is derived as follows ( $d 2$ is derived analogously):

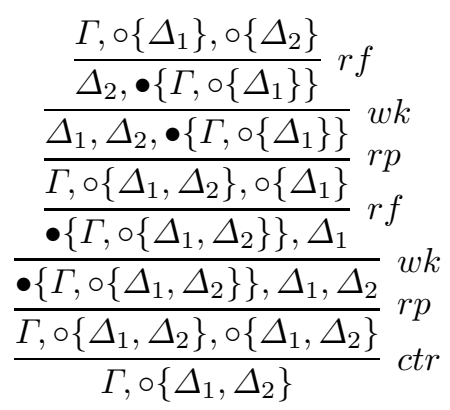

Lemma 16. Suppose $\vdash_{\mathrm{SKt}} \Pi_{1}: \Delta, \circ\{A\}$ and $\vdash_{\mathrm{SKt}} \Pi_{2}: \Sigma^{k}[\diamond \bar{A}]$, for some $k \geq 1$, and the cut ranks of $\Pi_{1}$ and $\Pi_{2}$ are smaller than $|\square A|$. Then there exists a proof $\Pi$ such that $\vdash_{\mathrm{SKt}} \Pi: \Sigma^{k}[\Delta]$ and $\operatorname{cr}(\Pi)<|\square A|$.

Proof. By induction on $\left|\Pi_{2}\right|$. The non-trivial case is when $\Pi_{2}$ ends with $\diamond$ on $\diamond \bar{A}$.

$$
\frac{\Sigma_{1}^{k-1}[\diamond \bar{A}], \circ\{\Gamma, \bar{A}\}}{\Sigma_{1}^{k-1}[\diamond \bar{A}], \circ\{\Gamma\}, \diamond \bar{A}} \diamond
$$

By induction hypothesis we have $\vdash_{\text {SKt }} \Pi^{\prime}: \Sigma_{1}^{k-1}[\Delta], \circ\{\Gamma, \bar{A}\}$ such that $\operatorname{cr}\left(\Pi^{\prime}\right)<$ $|\square A|$. The proof $\Pi$ is constructed as follows:

$$
\frac{\frac{\Sigma_{1}^{k-1}[\Delta], \circ\{\Gamma, \bar{A}\}}{\bullet\left\{\Sigma_{1}^{k-1}[\Delta]\right\}, \Gamma, \bar{A}} r f \quad \frac{\Delta, \circ\{A\}}{\bullet\{\Delta\}, A}}{\frac{\Pi_{1}}{\Pi^{\prime}}} r f
$$

Lemma 17. Suppose $\vdash_{\mathbf{S K t}} \Pi_{1}: \Delta, \circ\left\{\Delta^{\prime}, A\right\}$ and $\vdash_{\mathbf{S K t}} \Pi_{2}: \Sigma^{k}[\square \bar{A}]$, for some $k \geq 1$, and the cut ranks of $\Pi_{1}$ and $\Pi_{2}$ are smaller than $|\nabla A|$. Then there exists $\Pi$ such that $\vdash_{\mathbf{S K t}} \Pi: \Sigma^{k}\left[\Delta, \circ\left\{\Delta^{\prime}\right\}\right]$ and $\operatorname{cr}(\Pi)<|\diamond A|$.

Proof. By induction on $\left|\Pi_{2}\right|$. The non-trivial case: $\Pi_{2}$ is

$$
\frac{\sum_{1}^{k-1}[\square \bar{A}], \circ\{\bar{A}\}}{\Sigma_{1}^{k-1}[\square \bar{A}], \square \bar{A}}
$$

By induction hypothesis, we have

$$
\vdash \Pi^{\prime}: \Sigma_{1}^{k-1}\left[\Delta, \circ\left\{\Delta^{\prime}\right\}\right], \circ\{\bar{A}\}
$$


for some $\Pi^{\prime}$ such that $\operatorname{cr}\left(\Pi^{\prime}\right)<|\diamond A| . \Pi$ is constructed as follows:

$$
\frac{\frac{\sum_{1}^{k-1}\left[\Delta, \circ\left\{\Delta^{\prime}\right\}\right], \circ\{\bar{A}\}}{\bullet\left\{\Sigma_{1}^{k-1}\left[\Delta, \circ\left\{\Delta^{\prime}\right\}\right]\right\}, \bar{A}} r f \frac{\Delta, \circ\left\{\Delta^{\prime}, A\right\}}{\bullet\{\Delta\}, \Delta^{\prime}, A}}{\Pi_{1}} \mathrm{cut}
$$

Lemma 18. Suppose $\vdash_{\mathbf{S K t}} \Pi_{1}: \Delta, \bullet\{A\}$ and $\vdash_{\mathbf{S K t}} \Pi_{2}: \Sigma^{k}[\bar{A}]$, for some $k \geq 1$, and the cut ranks of $\Pi_{1}$ and $\Pi_{2}$ are smaller than $|A|$. Then there exists a proof $\Pi$ such that $\vdash_{\mathbf{S K t}} \Pi: \Sigma^{k}[\Delta]$ and $\operatorname{cr}(\Pi)<|\boldsymbol{\square} A|$.

Proof. This is proved analogously to Lemma 16.

Lemma 19. Suppose $\vdash_{\mathbf{S K t}} \Pi_{1}: \Delta, \bullet\left\{\Delta^{\prime}, A\right\}$ and $\vdash_{\mathbf{S K t}} \Pi_{2}: \Sigma^{k}[\overline{\mathbf{}} \bar{A}]$, for some $k \geq 1$, and the cut ranks of $\Pi_{1}$ and $\Pi_{2}$ are smaller than $|A|$. Then there exists $\Pi$ such that $\vdash_{\mathrm{SKt}} \Pi: \Sigma^{k}\left[\Delta, \bullet\left\{\Delta^{\prime}\right\}\right]$ and $\operatorname{cr}(\Pi)<|\vee A|$.

Proof. This is proved analogously to Lemma 17.

Lemma 1. Let $A$ be a non-atomic formula. Suppose $\vdash_{\mathbf{S K t}} \Pi_{1}: \Delta, \bar{A}$ and $\vdash_{\mathbf{S K t}}$ $\Pi_{2}: \Sigma^{k}[A]$, for some $k \geq 1$, and the cut ranks of $\Pi_{1}$ and $\Pi_{2}$ are smaller than $|A|$. Then there exists a proof $\Pi$ such that $\vdash_{\mathbf{S K t}} \Pi: \Sigma^{k}[\Delta]$ and $\operatorname{cr}(\Pi)<|A|$.

Proof. By induction on the height of $\Pi_{2}$ and case analysis on A. The nontrivial case is when $\Pi_{2}$ ends with an introduction rule on $A$. That is, we have $\Sigma^{k}[A]=\Sigma_{1}^{k-1}[A], A$ for some context $\Sigma_{1}^{k-1}[]$. We show the cases where $A$ is either $\square B, \diamond B$ or $B_{1} \wedge B_{2}$.

- Suppose $A=\square B$ and $\Pi_{2}$ is the following derivation:

$$
\begin{gathered}
\Pi_{2}^{\prime} \\
\frac{\sum_{1}^{k-1}[\square B], \circ\{B\}}{\sum_{1}^{k-1}[\square B], \square B} \square
\end{gathered}
$$

By induction hypothesis, we have $\vdash_{\mathbf{S K t}} \Pi^{\prime}: \Sigma_{1}^{k-1}[\Delta], \circ\{B\}$ and $\operatorname{cr}\left(\Pi^{\prime}\right)<$ $|A|$. Applying Lemma 16 to $\Pi^{\prime}$ and $\Pi_{1}$, we obtain $\vdash_{\text {SKt }} \Pi: \Delta, \Sigma_{1}^{k-1}[\Delta]=$ $\Sigma^{k}[\Delta]$ such that $\operatorname{cr}(\Pi)<|\square B|$.

- Suppose $A=\diamond B$ and $\Pi_{2}$ is the following derivation:

$$
\begin{aligned}
& \Pi_{2}^{\prime} \\
& \frac{\Sigma_{1}^{k-1}[\diamond B], \circ\left\{\Delta^{\prime}, B\right\}}{\Sigma_{1}^{k-1}[\diamond B], \circ\left\{\Delta^{\prime}\right\}, \diamond B} \diamond
\end{aligned}
$$

By induction hypothesis, we have $\vdash_{\mathbf{S K t}} \Pi^{\prime}: \Sigma_{1}^{k-1}[\Delta], \circ\left\{\Delta^{\prime}, B\right\}$. Applying Lemma 17 to $\Pi^{\prime}$ and $\Pi_{1}$, we obtain $\vdash_{\text {SKt }} \Pi: \Delta, \Sigma_{1}^{k-1}[\Delta]=\Sigma^{k}[\Delta]$ such that $\operatorname{cr}(\Pi)<|\diamond B|$. 
- Suppose $A=B_{1} \wedge B_{2}$ and $\Pi_{2}$ is the following derivation:

$$
\begin{array}{cc}
\Theta_{1} & \Theta_{2} \\
\frac{\Sigma_{1}^{k-1}\left[B_{1} \wedge B_{2}\right], B_{1}}{\Sigma_{1}^{k-1}\left[B_{1} \wedge B_{2}\right], B_{1} \wedge B_{2}} \Sigma_{1}^{k-1}\left[B_{1} \wedge B_{2}\right], B_{2}
\end{array}
$$

By induction hypothesis, we have $\vdash_{\mathbf{S K t}} \Theta_{1}^{\prime}: \Sigma_{1}^{k-1}[\Delta], B_{1}$ and $\vdash_{\mathbf{S K t}} \Theta_{2}^{\prime}$ : $\Sigma_{1}^{k-1}[\Delta], B_{2}$. Applying Lemma 14 to $\Theta_{1}^{\prime}, \Theta_{2}^{\prime}$ and $\Pi_{1}$, we obtain $\vdash_{\text {SKt }} \Pi$ : $\Delta, \Sigma_{1}^{k-1}[\Delta]=\Sigma^{k}[\Delta]$ such that $\operatorname{cr}(\Pi)<\left|B_{1} \wedge B_{2}\right|$.

Theorem 2. Cut elimination holds for SKt.

Proof. We remove topmost cuts in succession. Let $\Pi$ be a SKt-derivation with a topmost cut instance

$$
\begin{aligned}
& \Pi_{1} \quad \Pi_{2} \\
& \frac{\Gamma, A \quad \bar{A}, \Delta}{\Gamma, \Delta} \mathrm{cut}
\end{aligned}
$$

Note that $\Pi_{1}$ and $\Pi_{2}$ are both cut-free since this is a topmost instance in $\Pi$. We use induction on the size of $A$ to eliminate this topmost instance of cut. If $A$ is an atomic formula $a$ then we obtain a cut-free derivation $\Pi^{\prime}$ of $\Gamma, \Delta$ from applying Lemma 13 to $\Pi_{1}$ and $\Pi_{2}$.

If $A$ is non-atomic then we apply Lemma 1 to $\Pi_{2}$ and $\Pi_{1}$ and obtain a derivation $\Pi^{\prime}$ of $\Gamma, \Delta$ such that $\operatorname{cr}\left(\Pi^{\prime}\right)<|A|$. By the induction hypothesis, we can remove all the cuts in $\Pi^{\prime}$ to get a cut-free derivation of $\Gamma, \Delta$.

\section{B Proofs for Section 4}

Lemma 2 (Admissibility of weakening). Suppose $\vdash_{\text {DKt }} \Pi: \Sigma[\Gamma]$. Then for every $\Delta$, there exists $\Pi^{\prime}$ such that $\vdash_{\mathrm{DKt}} \Pi^{\prime}: \Sigma[\Gamma, \Delta]$ and $\left|\Pi^{\prime}\right| \leq|\Pi|$.

Proof. By simple induction on $|\Pi|$.

Lemma 3 (Admissibility of display postulates). Suppose $\vdash_{\mathrm{DKt}} \Pi$ : $\Gamma, \bullet\{\Delta\}$. Then there exists $\Pi^{\prime}$ such that $\vdash_{\text {DKt }} \Pi^{\prime}: \circ\{\Gamma\}, \Delta$ such that $\left|\Pi^{\prime}\right| \leq|\Pi|$.

Proof. By induction on $|\Pi|$. The non-trivial cases are when there is an exchange of formulae between $\Gamma$ and $\Delta$. One example is as follows: Suppose $\Pi$ is

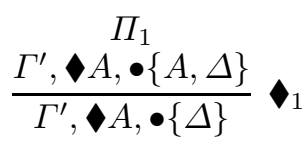

Then $\Pi^{\prime}$ is

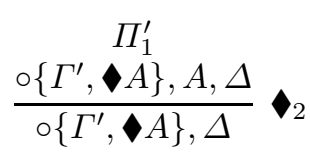

where $\Pi_{1}^{\prime}$ is obtained from induction hypothesis. 
Lemma 4 (Admissibility of display postulates). Suppose $\vdash_{\mathrm{DKt}} \Pi$ : $\Gamma, \circ\{\Delta\}$. Then there exists $\Pi^{\prime}$ such that $\vdash_{\mathbf{D K t}} \Pi^{\prime}: \bullet\{\Gamma\}, \Delta$ such that $\left|\Pi^{\prime}\right| \leq|\Pi|$.

Proof. Analogous to the proof of Lemma 3.

To show admissibility of contraction, we first need to show certain distributivity properties, stated in the following two lemmas, and admissibility of formula contraction.

Lemma 20. If $\vdash_{\text {DKt }} \Pi: \Sigma\left[\circ\left\{\Delta_{1}\right\}, \circ\left\{\Delta_{2}\right\}\right]$ then there exists $\Pi^{\prime}$ such that $\vdash_{\mathbf{D K t}}$ $\Pi^{\prime}: \Sigma\left[\circ\left\{\Delta_{1}, \Delta_{2}\right\}\right]$ and $\left|\Pi^{\prime}\right| \leq|\Pi|$.

Proof. By induction on $|\Pi|$. We show here two non-trivial cases:

- Suppose $\Pi$ ends with $\diamond_{1}$ that moves a formula into $\circ\left\{\Delta_{1}\right\}$. That is,

$$
\Sigma\left[\circ\left\{\Delta_{1}\right\}, \circ\left\{\Delta_{2}\right\}\right]=\Sigma^{\prime}\left[\diamond A, \circ\left\{\Delta_{1}\right\}, \circ\left\{\Delta_{2}\right\}\right]
$$

and $\Pi$ is

$$
\frac{\Sigma^{\prime}\left[\diamond A, \circ\left\{A, \Delta_{1}\right\}, \circ\left\{\Delta_{2}\right\}\right]}{\Sigma^{\prime}\left[\diamond A, \circ\left\{\Delta_{1}\right\}, \circ\left\{\Delta_{2}\right\}\right]} \diamond_{1}
$$

By induction hypothesis, we have a proof $\Psi^{\prime}$ such that

$$
\vdash_{\text {DKt }} \Psi^{\prime}: \Sigma^{\prime}\left[\diamond A, \circ\left\{A, \Delta_{1}, \Delta_{2}\right\}\right]
$$

and $\left|\Psi^{\prime}\right| \leq|\Psi|<|\Pi|$. The proof $\Pi^{\prime}$ is constructed as follows:

$$
\frac{\Sigma^{\prime}\left[\nabla A, \circ\left\{A, \Delta_{1}, \Delta_{2}\right\}\right]}{\Sigma^{\prime}\left[\diamond A, \circ\left\{\Delta_{1}, \Delta_{2}\right\}\right]} \diamond_{1}
$$

- Suppose $\Pi$ ends with ${ }_{2}$ that moves a formula out from $\circ\left\{\Delta_{1}\right\}$. That is, $\Delta_{1}=A, \Delta_{1}^{\prime}$ and $\Pi$ is

$$
\frac{\Sigma\left[A, \circ\left\{A, \Delta_{1}^{\prime}\right\}, \circ\left\{\Delta_{2}\right\}\right]}{\Sigma\left[\circ\left\{\triangleleft, \Delta_{1}^{\prime}\right\}, \circ\left\{\Delta_{2}\right\}\right]} \diamond_{2}
$$

Then $\Pi^{\prime}$ is constructed as follows

$$
\frac{\Sigma\left[A, \circ\left\{\Psi^{\prime}\right.\right.}{\Sigma\left[\circ\left\{\Delta_{1}, \Delta_{2}\right\}\right]} \bullet_{2}
$$

where $\Psi^{\prime}$ is obtained from the induction hypothesis. It is easy to see that $\left|\Pi^{\prime}\right| \leq|\Pi|$.

Lemma 21. If $\vdash_{\text {DKt }} \Pi: \Sigma\left[\bullet\left\{\Delta_{1}\right\}, \bullet\left\{\Delta_{2}\right\}\right]$ then there exists $\Pi^{\prime}$ such that $\vdash_{\mathbf{D K t}}$ $\Pi^{\prime}: \Sigma\left[\bullet\left\{\Delta_{1}, \Delta_{2}\right\}\right]$ and $\left|\Pi^{\prime}\right| \leq|\Pi|$. 
Proof. Analogous to the proof of Lemma 20.

Lemma 22. If $\vdash_{\text {DKt }} \Pi: \Sigma[A, A]$ then there exists $\Pi^{\prime}$ such that $\vdash_{\mathbf{D K t}} \Pi^{\prime}: \Sigma[A]$ and $\left|\Pi^{\prime}\right| \leq|\Pi|$.

Proof. Straightforward by induction on $\Pi \mid$.

Lemma 5 (Admissibility of contraction). If $\vdash_{\text {DKt }} \Pi: \Sigma[\Delta, \Delta]$ then there exists $\Pi^{\prime}$ such that $\vdash_{\mathbf{D K t}} \Pi^{\prime}: \Sigma[\Delta]$ and $\left|\Pi^{\prime}\right| \leq|\Pi|$.

Proof. By induction on the size of $\Delta$, Lemma 20, Lemma 21, and Lemma 22. If $\Delta$ is a singleton set containing one formula, then the lemma follows immediately from Lemma 22 . The other cases follow from the induction hypothesis and Lemma 20 and Lemma 21. Consider, for instance, the case where $\Delta=\circ\left\{\Delta^{\prime}\right\}$. Then by Lemma 20 we have a proof $\Psi$ such that

$$
\vdash_{\text {DKt }} \Psi: \Sigma\left[\circ\left\{\Delta^{\prime}, \Delta^{\prime}\right\}\right]
$$

and $|\Psi| \leq|\Pi|$. Note that since $\Delta^{\prime}$ is of a smaller size than $\circ\left\{\Delta^{\prime}\right\}$, we can apply the induction hypothesis to $\Psi$ and obtain a proof $\Pi^{\prime}$ such that

$$
\vdash_{\mathrm{DKt}} \Pi^{\prime}: \Sigma\left[\circ\left\{\Delta^{\prime}\right\}\right]
$$

and $\left|\Pi^{\prime}\right| \leq|\Pi|$.

Theorem 3. For every sequent $\Gamma, \vdash_{\mathbf{S K t}} \Gamma$ if and only if $\vdash_{\mathbf{D K t}} \Gamma$.

Proof. The forward direction, that is, showing that $\vdash_{\mathbf{S K t}} \Gamma$ implies $\vdash_{\text {DKt }} \Gamma$, follows from admissibility of the structural rules of SKt in DKt (Lemma 2 Lemma 5).

For the converse, we use the display property of SKt (Proposition 1) to simulate the deep-inference rules of DKt. We show here the derivations for the rules $\nabla_{1}$ and $\nabla_{2}$ (the other cases are similar):

$$
\begin{aligned}
& \Sigma[\circ\{\Delta, \diamond A\}, A] \\
& \Sigma[\circ\{\Delta, A\}, \diamond A] \\
& \frac{\frac{\Delta^{\prime}, \circ\{\Delta, A\}, \diamond A}{\Delta^{\prime}, \circ\{\Delta\}, \diamond A, \diamond A}}{\Delta^{\prime}, \circ\{\Delta\}, \diamond A} c t r
\end{aligned}
$$

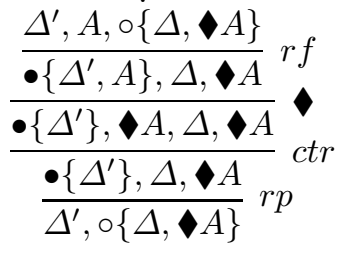

$$
\begin{aligned}
& \Sigma[\circ\{\dot{\Delta}\}, \diamond A] \\
& \Sigma[\circ\{\dot{\Delta}, \diamond A\}]
\end{aligned}
$$

where the dots part of the derivation is obtained from applying Proposition 1.

Theorem 4 (Separation). For every modal formula $\varphi, \vdash_{\mathbf{D K}} \varphi$ iff $\varphi$ is a theorem of $\mathrm{K}$. 
Proof. $(\Rightarrow)$ Suppose $\vdash_{\mathbf{D K}} \varphi$. Since $\mathbf{D K}$ is a subsystem of DKt, we must have $\vdash_{\text {DKt }} \varphi$, and then $\vdash_{\text {SKt }} \varphi$. By the soundness of SKt, $\varphi$ is Kt-valid. But all purely modal $K t$-valid formulae are also $K$-valid. Thus purely modal $\varphi$ is also a theorem of $\mathrm{K}$.

$(\Leftarrow)$ Suppose $\varphi$ is a theorem of K. But the theorems of K are also theorems of Kt, hence $\varphi$ is derivable in SKt. This derivation may contain cuts, but by cutelimination we know that $\varphi$ is also cut-free derivable in $\mathbf{S K t}$. The cut-free $\mathbf{S K t}$ derivation of a purely modal formula cannot contain any instances of the rules - or since these introduce non-modal connectives into their conclusion. Thus, the only way to create an occurrence of $\bullet$ on our way up from the end-sequent is to use $r p$. By Theorem 3, the cut-free SKt-derivation of $\varphi$ can be transformed into a (cut-free) derivation of $\varphi$ in DKt. Moreover, the transformation given in the proof removes all applications of $r p$ without creating black structural or logical connectives. For example, an SKt derivation of $a, \bar{a}, \bullet\{\Delta\}$ is converted to a DKt derivation of $(\circ\{a, \bar{a}\}, \Delta)=\Sigma[a, \bar{a}]$. Hence the transformed derivation is actually a derivation in DK.

\section{Proofs for Section 5}

\section{C.1 Modal Tense Logic S4}

Lemma 6. Every rule of DS4 is derivable in SS4.

Proof. We show here derivations of $T_{a}$ and $4_{a}$; the others are not difficult. In the derivations below, double lines indicate multiple applications of the residuation rules $r p$ and/or $r f$, according to Proposition 1.

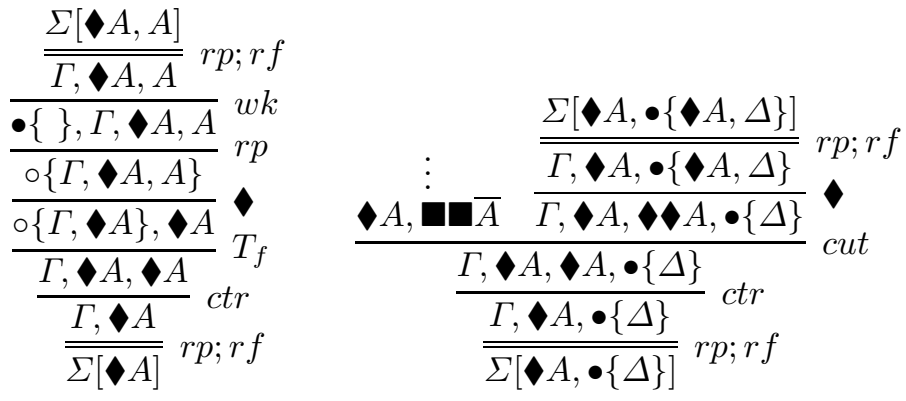

Note that the sequent $A, \mathbf{\square} \bar{A}$ in the derivation above right can easily be shown provable in SS4.

Lemma 23 (Admissibility of $T_{f}$ ). Suppose $\vdash_{\mathrm{DS} 4} \Pi: \Sigma[\circ\{\Delta\}]$. Then there exists $\Pi^{\prime}$ such that $\vdash_{\mathbf{D S} 4} \Pi^{\prime}: \Sigma[\Delta]$.

Proof. The non-trivial case is when $\Pi$ ends with a diamond-rule that moves in or out of $\circ\{\Delta\}$. 
- Suppose $\Pi$ is

$$
\begin{gathered}
\Pi_{1} \\
\frac{\Sigma^{\prime}[\circ\{\Delta, A\}, \diamond A]}{\Sigma^{\prime}[\circ\{\Delta\}, \diamond A]} \diamond_{1}
\end{gathered}
$$

By induction hypothesis: there exists $\Pi_{1}^{\prime}$ s.t.

$$
\vdash_{\mathrm{DS} 4} \Pi_{1}^{\prime}: \Sigma^{\prime}[\Delta, A, \diamond A] .
$$

Then $\Pi^{\prime}$ is constructed as follows:

$$
\begin{gathered}
\Pi_{1}^{\prime} \\
\frac{\Sigma^{\prime}[\Delta, A, \diamond A]}{\Sigma^{\prime}[\Delta, \diamond A]} T_{b}
\end{gathered}
$$

- The case where $\Pi$ ends with

$$
\begin{array}{ccc}
\Pi_{1} & \Pi_{1}^{\prime} \\
\frac{\Sigma\left[\circ\left\{\Delta^{\prime}, \diamond A\right\}, A\right]}{\Sigma\left[\circ\left\{\Delta^{\prime}, \triangleleft A\right\}\right]} \diamond_{2} \Longrightarrow & \Longrightarrow & \frac{\Sigma\left[\Delta^{\prime}, \diamond A, A\right]}{\Sigma\left[\Delta^{\prime}, \diamond A\right]} T_{a}
\end{array}
$$

- $\Pi$ ends with $4_{c}$ :

$$
\begin{gathered}
\Pi_{1} \\
\frac{\Sigma^{\prime}[\nabla A, \circ\{\diamond A, \Delta\}]}{\Sigma^{\prime}[\diamond A, \circ\{\Delta\}]} 4_{c}
\end{gathered}
$$

By induction hypothesis, we have

$$
\vdash_{\mathrm{DS} 4} \Pi_{1}^{\prime}: \Sigma^{\prime}[\diamond A, \diamond A, \Delta] .
$$

Now apply the height-preserving admissibility lemma for contraction, and we have $\vdash \Pi^{\prime}: \Sigma^{\prime}[\diamond A, \Delta]$.

The other cases involving axiom 4 can be done similarly.

Lemma 24 (Admissibility of $T_{p}$ ). Suppose $\vdash_{\mathrm{DS} 4} \Pi: \Sigma[\bullet\{\Delta\}]$. Then there exists $\Pi^{\prime}$ such that $\vdash_{\mathrm{DS} 4} \Pi^{\prime}: \Sigma[\Delta]$.

Proof. Analogous to the proof of Lemma 23.

Lemma 25 (Admissibility of $4_{f}$ ). Suppose $\vdash_{\mathrm{DS} 4} \Pi: \Sigma[\circ\{\Delta\}]$. Then there exists $\Pi^{\prime}$ such that $\vdash_{\mathbf{D S} 4} \Pi^{\prime}: \Sigma[\circ\{\circ\{\Delta\}\}]$.

Proof. The non-trivial cases are when a diamond formula moves in or out of $\circ\{\Delta\}$.

- Suppose $\Pi$ is

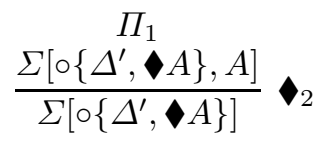


By induction hypothesis, we have

$$
\vdash \Pi_{1}^{\prime}: \Sigma\left[\circ\left\{\circ\left\{\Delta^{\prime}, \diamond A\right\}\right\}, A\right] .
$$

Apply the weakening lemma to get

$$
\vdash \Pi_{2}: \Sigma\left[\circ\left\{\circ\left\{\Delta^{\prime}, \diamond A\right\}, \diamond A\right\}, A\right] .
$$

Then $\Pi^{\prime}$ is constructed as follows:

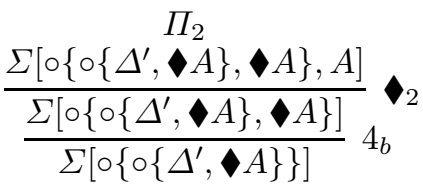

- Suppose $\Pi$ is

$$
\begin{aligned}
& \Pi_{1} \\
& \frac{\Sigma^{\prime}[\circ\{\Delta, A\}, \diamond A]}{\Sigma^{\prime}[\circ\{\Delta\}, \diamond A]} \diamond_{1}
\end{aligned}
$$

By induction hypothesis, we have

$$
\vdash \Pi_{1}^{\prime}: \Sigma^{\prime}[\circ\{\circ\{\Delta, A\}\}, \diamond A]
$$

Apply the weakening lemma, we have

$$
\vdash \Pi_{2}: \Sigma^{\prime}[\circ\{\circ\{\Delta, A\}, \diamond A\}, \diamond A]
$$

Now $\Pi^{\prime}$ is constructed as follows:

$$
\frac{\Sigma^{\prime}[\circ\{\circ\{\Delta, A\}, \diamond A\}, \diamond A]}{\frac{\Pi_{2}}{\Sigma^{\prime}[\circ\{\circ\{\Delta\}, \diamond A\}, \diamond A]}} \diamond_{1}
$$

- The other cases can be proved similarly.

Lemma 26 (Admissibility of $4_{p}$ ). Suppose $\vdash_{\mathbf{D S} 4} \Pi: \Sigma[\bullet\{\Delta\}]$. Then there exists $\Pi^{\prime}$ such that $\vdash_{\mathbf{D S} 4} \Pi^{\prime}: \Sigma[\bullet\{\bullet\{\Delta\}\}]$.

Proof. Analogous to the proof of Lemma 25.

\section{C.2 Modal tense logic S5}

Lemma 27 (Admissibility of $B_{1}$ ). Suppose $\vdash_{\text {Ds5 }} \Pi: \Sigma[\circ\{\Delta\}]$. Then there exists $\Pi^{\prime}$ such that $\vdash_{\text {DS5 }} \Pi^{\prime}: \Sigma[\bullet\{\Delta\}]$.

Proof. The non-trivial cases are when $\Pi$ ends with a diamond-rule that moves in or out of $\circ\{\Delta\}$. 
- Suppose $\Pi$ is

$$
\begin{aligned}
& \Pi_{1} \\
& \frac{\Sigma^{\prime}\left[\circ\left\{\Delta^{\prime}, A\right\}, \diamond A\right]}{\Sigma^{\prime}\left[\circ\left\{\Delta^{\prime}\right\}, \diamond A\right]} \diamond_{1}
\end{aligned}
$$

By induction hypothesis, we have

$$
\vdash_{\mathrm{DS} 5} \Pi_{1}^{\prime}: \Sigma^{\prime}\left[\bullet\left\{\Delta^{\prime}, A\right\}, \diamond A\right] .
$$

By weakening, we have

$$
\vdash_{\mathrm{DS5}} \Pi_{2}: \Sigma^{\prime}\left[\bullet\left\{\Delta^{\prime}, A, \diamond A\right\}, \diamond A\right] .
$$

Now $\Pi^{\prime}$ is constructed as follows:

$$
\begin{aligned}
& \Pi_{2}
\end{aligned}
$$

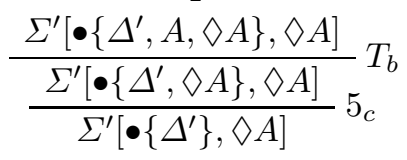

- Suppose $\Pi$ is

$$
\begin{aligned}
& \Pi_{1} \\
& \frac{\Sigma^{\prime}\left[\circ\left\{\Delta^{\prime}, A\right\}, A\right]}{\Sigma^{\prime}\left[\circ\left\{\Delta^{\prime}, A\right\}\right]} \diamond_{2}
\end{aligned}
$$

By induction hypothesis, we have

$$
\vdash_{\mathrm{DS} 5} \Pi_{1}^{\prime}: \Sigma^{\prime}\left[\bullet\left\{\Delta^{\prime}, \diamond A\right\}, A\right] .
$$

By weakening, we have

$$
\vdash_{\mathrm{DS5}} \Pi_{2}: \Sigma^{\prime}\left[\bullet\left\{\Delta^{\prime}, \diamond A\right\}, A, \diamond A\right] .
$$

Now $\Pi^{\prime}$ is constructed as follows:

$$
\begin{aligned}
& \Pi_{2}
\end{aligned}
$$

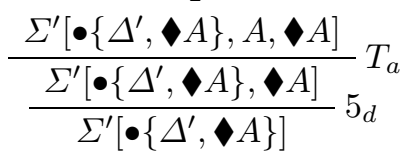

- Suppose $\Pi$ is

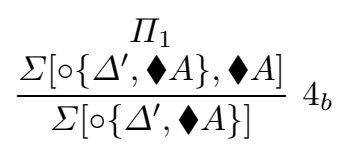

Then $\Pi^{\prime}$ is constructed as follows:

$$
\begin{aligned}
& \Pi_{1}^{\prime} \\
& \frac{\Sigma\left[\bullet\left\{\Delta^{\prime}, \bullet A\right\}, \bullet A\right]}{\Sigma\left[\bullet\left\{\Delta^{\prime}, \bullet A\right\}\right]} 5_{d}
\end{aligned}
$$

where $\Pi_{1}^{\prime}$ is obtained by applying the induction hypothesis to $\Pi_{1}$. 
- Suppose $\Pi$ is

$$
\begin{gathered}
\Pi_{1} \\
\frac{\Sigma^{\prime}[\diamond A, \circ\{\diamond A, \Delta\}]}{\Sigma^{\prime}[\diamond A, \circ\{\Delta\}]} 4_{c}
\end{gathered}
$$

Then $\Pi^{\prime}$ is constructed as follows:

$$
\begin{gathered}
\Pi_{1}^{\prime} \\
\frac{\Sigma[\nabla A, \bullet\{\diamond A, \Delta\}]}{\Sigma^{\prime}[\nabla A, \bullet\{\Delta\}]} 5_{c}
\end{gathered}
$$

where $\Pi_{1}^{\prime}$ is obtained by applying the induction hypothesis to $\Pi_{1}$.

- Suppose $\Pi$ is

$$
\begin{aligned}
& \Pi_{1} \\
& \frac{\Sigma^{\prime}\left[\bullet A, \circ\left\{\bullet A, \Delta^{\prime}\right\}\right]}{\Sigma^{\prime}\left[\bullet A, \circ\left\{\Delta^{\prime}\right\}\right]} 5_{a}
\end{aligned}
$$

By induction hypothesis, we have

$$
\vdash_{\text {DS5 }} \Pi_{1}^{\prime}: \Sigma^{\prime}\left[\triangleleft A, \bullet\left\{\triangleleft A, \Delta^{\prime}\right\}\right] .
$$

Now $\Pi^{\prime}$ is constructed as follows:

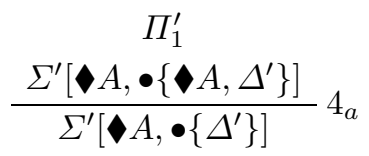

- Suppose $\Pi$ is

$$
\begin{aligned}
& \Pi_{1} \\
& \frac{\Sigma^{\prime}\left[\circ\left\{\Delta^{\prime}, \diamond A\right\}, \diamond A\right]}{\Sigma^{\prime}\left[\circ\left\{\Delta^{\prime}, \diamond A\right\}\right]} 5_{b}
\end{aligned}
$$

By induction hypothesis, we have

$$
\vdash_{\text {DS5 }} \Pi_{1}^{\prime}: \Sigma^{\prime}\left[\bullet\left\{\Delta^{\prime}, \diamond A\right\}, \diamond A\right] .
$$

Now $\Pi^{\prime}$ is constructed as follows:

$$
\begin{aligned}
& \Pi_{1}^{\prime} \\
& \frac{\Sigma^{\prime}\left[\bullet\left\{\Delta^{\prime}, \diamond A\right\}, \diamond A\right]}{\Sigma^{\prime}\left[\bullet\left\{\Delta^{\prime}, \diamond A\right\}\right.} 4_{d}
\end{aligned}
$$

Lemma 28 (Admissibility of $B_{2}$ ). Suppose $\vdash_{\mathrm{DS} 5} \Pi: \Sigma[\bullet\{\Delta\}]$. Then there exists $\Pi^{\prime}$ such that $\vdash_{\mathrm{DS} 5} \Pi^{\prime}: \Sigma[0\{\Delta\}]$.

Proof. Analogous to the proof of Lemma 27. 


\section{Proofs for Section 6}

Theorem 12. Function Prove terminates for any input sequent $\Xi$.

Proof. Let $m=|s f(\Xi)|, d=\operatorname{deg}(s f(\Xi)) \leq m$ and $T=\operatorname{tree}(\Xi)$.

The saturation process for each node in $T$ is bounded by $m$. Therefore after at most $m$ moves at each node, Step 3 is no longer applicable to this node.

$T$ is finitely branching, since new nodes are only created for unrealised box formulae. Therefore after at most $m$ moves at each node, Step 4 is no longer applicable to this node. The depth of $T$ is bounded by $d$, since each node $\Theta \in T$ at distance $k$ from the root of $T$ has degree $(\Theta) \leq d-k$.

Since $\diamond$ - and $\diamond$-prefixed formulae are only propagated to nodes that do not already contain these formulae, after at most $m$ propagation moves into each node, Step 5 is no longer applicable to this node.

Lemma 29 (Admissibility of $\diamond_{1}$ ). Suppose $\vdash_{\mathrm{DS} 4} \Pi: \Sigma[\circ\{\Delta, A\}, \diamond A]$. Then there exists $\Pi^{\prime}$ such that $\vdash_{\mathbf{D s} \mathbf{4}^{-}} \Pi^{\prime}: \Sigma[\circ\{\Delta\}, \diamond A]$.

Proof. By the weakening lemma, $\vdash_{\mathbf{D S}^{-}} \Pi_{1}: \Sigma[\circ\{\Delta, \diamond A, A\}, \diamond A]$. Then the following gives us the required derivation:

$$
\begin{aligned}
& \Pi_{1}
\end{aligned}
$$

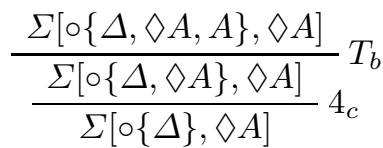

Lemma 30 (Admissibility of $\diamond_{2}$ ). Suppose $\vdash_{\text {DS4 }} \Pi: \Sigma[\bullet\{\Delta, \diamond A\}, A]$. Then there exists $\Pi^{\prime}$ such that $\vdash_{\mathbf{D S} \mathbf{4}^{-}} \Pi^{\prime}: \Sigma[\bullet\{\Delta, \diamond A\}]$.

Proof. By the weakening lemma, $\vdash_{\mathbf{D S}_{4^{-}}} \Pi_{1}: \Sigma[\bullet\{\Delta, \diamond A\}, \diamond A, A]$. Then the following gives us the required derivation:

$$
\begin{aligned}
& \Pi_{1}
\end{aligned}
$$

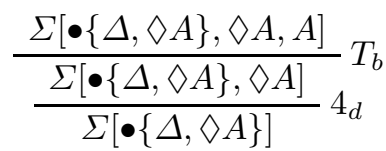

Lemma 31 (Admissibility of $\diamond_{1}$ ). Suppose $\vdash_{\text {DS4 }} \Pi: \Sigma[\bullet\{\Delta, A\}, \diamond A]$. Then there exists $\Pi^{\prime}$ such that $\vdash_{\mathbf{D s} \mathbf{4}^{-}} \Pi^{\prime}: \Sigma[\bullet\{\Delta\}, \diamond A]$.

Proof. By the weakening lemma, $\vdash_{\mathbf{D S 4}^{-}} \Pi_{1}: \Sigma[\bullet\{\Delta, \diamond A, A\}, \triangleleft A]$. Then the following gives us the required derivation:

$$
\begin{aligned}
& \Pi_{1}
\end{aligned}
$$

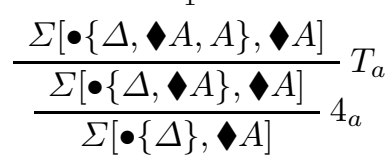


Lemma 32 (Admissibility of $\diamond_{2}$ ). Suppose $\vdash_{\mathrm{DS} 4} \Pi: \Sigma[\circ\{\Delta, \diamond A\}, A]$. Then there exists $\Pi^{\prime}$ such that $\vdash_{\mathbf{D s} \mathbf{4}^{-}} \Pi^{\prime}: \Sigma[\circ\{\Delta, \diamond A\}]$.

Proof. By the weakening lemma, $\vdash_{\mathbf{D S} 4^{-}} \Pi_{1}: \Sigma[\circ\{\Delta, \diamond A\}, \triangleleft A, A]$. Then the following gives us the required derivation:

$$
\begin{aligned}
& \Pi_{1}
\end{aligned}
$$

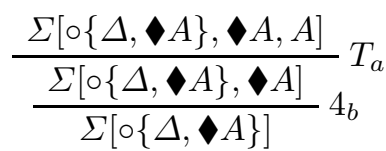

Lemma 33. For every $\mathbf{D S} 4^{*}$-derivation $\Pi$, for every sequent $\Xi \in \Pi$, the maximum number of consecutive o-edges in tree $(\Xi)$ is $m^{2}$, where $m=|s f(\Xi)|$.

Proof. A o-edge can only be created by a $\square$-rule application. After at most $m$ consecutive $\square$-rule applications, the $\square$ rule will be blocked because every $\square$-formula will be tagged. The $\square$ rule can only become applicable again if the tagged formulae are removed (i.e., the history is cleared) by a $T_{b}^{1}$ rule application. After at most $m T_{b}^{1}$ rule applications, the $T_{b}^{1}$ rule will be blocked because every $\diamond$-formula will be tagged. 